\title{
An adaptive q-Lognormal model towards the computation of Average Channel Capacity in slow fading channels
}

Tanmay Mukherjee ( $\square$ utanmay10@gmail.com )

Ravenshaw University https://orcid.org/0000-0003-1290-0703

Dilip Senapati

Ravenshaw University https://orcid.org/0000-0002-3157-4627

\section{Research Article}

Keywords: Tsallis' entropy, Shadowing, q-Lognormal, JS measure, Adaptability, Monte-Carlo Simulation, Gauss-Hypergeometric function

Posted Date: March 24th, 2021

DOl: https://doi.org/10.21203/rs.3.rs-223949/v1

License: (c) (i) This work is licensed under a Creative Commons Attribution 4.0 International License.

Read Full License

Version of Record: A version of this preprint was published at Telecommunication Systems on January 4th, 2022. See the published version at https://doi.org/10.1007/s11235-021-00843-5. 
Noname manuscript No.

(will be inserted by the editor)

An adaptive $q$-Lognormal model towards the computation of Average Channel Capacity in slow fading channels

Tanmay Mukherjee · Dilip Senapati

Received: date / Accepted: date

Tanmay Mukherjee

Department of Computer Science

Ravenshaw University

Odisha-753003,India

Tel.: +191-7735003495

E-mail: utanmay10@gmail.com

Corresponding Author: Dr. Dilip Senapati

Department of Computer Science,

Ravenshaw University, Odisha, India.

Email: senapatidillip@gmail.com

Telephone: +91-7377948428 


\begin{abstract}
The characterization of multipath fading and shadowing in wireless communication systems is essential towards the evaluation of various performance measures. It is well known that the statistical characterization of shadowing phenomena is captured by distributions viz., log-normal distribution, gamma distribution and other mixture distributions. However, it is observed that the log-normal distribution fails to characterize the outliers in the fading signal. The extreme fluctuations in the fading signal needs to be characterized efficiently for error free computation of the various performance metrics. In this context, this paper portrays an adaptive generalized Tsallis' non-extensive ' $q$ '-Lognormal model towards the characterization of various fading channels. This model operates well with the synthesized fading signals and captures the wide range of tail fluctuations to adapt different fading scenarios. The significance and applicability of the proposed novel $q$-lognormal model in capturing the slow fading channels is validated using different statistical tests viz., chisquare test and symmetric JS measure. Furthermore, essential performance measures viz., the average channel capacity, closed form expression of cumulative distribution function $(\mathrm{CDF})$ in terms of Gauss-Hypergeometric function ${ }_{2} F_{1}[\mathrm{a}, \mathrm{b}, \mathrm{c} ; \mathrm{z}]$, higher order moments corresponding to ' $q$ '-Lognormal channel capacity and coefficient of variation is evaluated corresponding to the proposed $q$-lognormal model performing extensive Monte-Carlo simulation techniques up to $O\left(10^{7}\right)$.
\end{abstract}

Keywords Tsallis' entropy · Shadowing · $q$-Lognormal · JS measure · Adaptability · Monte-Carlo Simulation · Gauss-Hypergeometric function

\title{
1 Introduction
}

In pragmatic wireless propagation environments, the channels gets impaired by shadowing phenomena. It arises in conditions when the coherence time of the transmission channel is large corresponding to the delay constraint of the channel $[1,5]$. The shadowing effects will result in the signals bending i.e., the transmitted signals will not follow a straight propagation path. In this arrangement, the phase and amplitude variations exploited over the communication channel could be contemplated roughly constant throughout the period of its existence. The amplitude fluctuations as a result of shadowing is often modeled using the log-normal distribution with the fluctuation parameter satisfying the log distance path loss model $[2,3,15]$. The log-normal distribution is often averaged with several other distributions viz., the Rayleigh distribution, the Weibull distribution to contemplate the concurrent effects of fading and shadowing $[23,18,27]$. It is observed that the log-normal distribution has an ascendancy over other slow fading channel models viz., gamma distribution, inverse gaussian distribution $[4,16]$ in capturing the long range fading signals. However, the widely used conventional log-normal model fails to capture the extreme fluctuations due to its inability in distinguishing the tail behavior or outliers of the fading signals[23,27]. The outliers are the regions in the signal 
where the extreme fluctuations are observed due to various obstructions between the transmitter and the receiver, and this should be analyzed effectively for error free signal transmission and evaluation of performance measures.

In this context, a framework incorporating maximum entropy principle based on non-extensive parameter ' $q$ ' defined by Tsallis' is portrayed to explain the extreme tail fluctuations of the fading signals $[10,9,14,28,7]$. The specification of the normalization constraint along with first and second moment constraints results in the $q$-Lognormal model [23] for the continuous range of parameter ' $q$ ', $1<q<3$. The presented $q$-Lognormal model can characterize an entire scale of tail variations in the real valued range of non-extensive parameter ' $q$ ', and the model adapts to different fading scenarios. When $q \rightarrow 1$, the proposed model mimics the conventional log-normal distribution. In Tsallis entropy framework, the value of the non-extensive parameter $q$ between the proposed $q$-Lognormal distribution and synthesized fading data is estimated by optimizing the generic Jensen-Shannon (JS) divergence. In contrary to the commonly modeled lognormal distribution, as $q>1$ the proposed model portrays the long-tailed characteristics that provides an excellent agreement over the outliers in the fading signals [23].

The evaluation of channel capacity over $q$-lognormal fading is an important performance criterion for the estimation of the maximum transmission capacity that can be reached by a communication channel with an arbitrary low probability of error $[19,21,22]$. In various fading situations, a wide range of theories concerning channel capability have been portrayed in literature. As defined in IEEE 802.15.3a wireless communication system, the lognormal probability density function (PDF) well characterizes small scale fading [24]. In [25], the lower and upper range have been portrayed corresponding to average channel capacity but in case of high decibel $(\mathrm{dB})$ spread these appear to be in weak agreement. When the transmission time is sufficiently long, stretching over a large number of coherence periods and thus achieving long-term ergodicity in the fading process, the term ergodic channel capacity (Shannon capacity) is used [19]. Moreover, this paper presents the theoretical model for the ergodic channel capacity over the proposed $q$-lognormal distribution. However, it is interesting to observe that the theoretical derivation of CDF corresponding to the channel capacity model with respect to $q$-Lognormal model is obtained in terms of well-known Gauss Hypergeometric function, ${ }_{2} F_{1}$ [a, b, c; z].

In this paper, the contribution of the presented $q$-Lognormal model in contrast to the well-known conventional log-normal model has been portrayed to characterize the shadowing environment in wireless communications. Moreover, to validate the proposed model and its adaptability in slow fading channels, different statistical tests viz., chi-square test and generalized symmetric JS measure have been tested. The remainder of the paper goes on as follows. In Section 2, the probability density function (PDF) of the proposed $q$-Lognormal model is expressed in closed form. Section 3, deals with the evaluation of goodness of fit and the relative error of the proposed model in contrast to the conventional log-normal model. This section also presents a table of mathematical notations and functions used throughout the paper. In 
Section 4, the generalized symmetric JS metric corresponding to theoretical and synthesized data is portrayed . The closed form solutions corresponding to the PDF and CDF of the average channel capacity in $q$-lognormal fading scenarios is derived in Section 5. In Section $6, K^{\text {th }}$ order moments of lognormal model and the proposed $q$-Lognormal model for slow fading channels have been portrayed and validated using Monte-Carlo simulation schemes. In Section 7, an important statistical measure i.e., Coefficient of Variation (CV) is evaluated corresponding to the proposed model. The final Section 8 includes the conclusion of the work.

\section{$2 q$-Lognormal probability density function (PDF)}

This section provides the formulation procedure of non-extensive $q$-Lognormal PDF. The desired PDF undergoes the difference of integral sign of the corresponding Lagrangian function, which is constructed following maximum entropy principle i.e., non-extensive $q$-Lognormal PDF is maximized Tsallis' entropy with normalized, first, and second moment constrains [10,9].

Let $\Gamma$ denote the random variable of the received average received Signal to Noise ratio (SNR) $(\gamma)$ i.e.,

$$
\Gamma \equiv \ln (\gamma), \quad \gamma>0 .
$$

The expression for Tsallis' entropy incorporating the parameter ' $q$ ' $[9,10]$ is given as

$$
T_{q}(\gamma)=\frac{1-\int_{-\infty}^{+\infty}\left[\varphi_{q}(\gamma)\right]^{q} d \gamma}{q-1} .
$$

The normalization constant, first and second moment of SNR are given as

$$
\begin{aligned}
& \int_{-\infty}^{+\infty} \varphi_{q}(\gamma) d \gamma=1 \\
& \int_{-\infty}^{+\infty} \varphi_{e s}(\gamma) \gamma d \gamma=\mu
\end{aligned}
$$

and

$$
\int_{-\infty}^{+\infty} \varphi_{e s}(\gamma)(\gamma-\mu)^{2} d \gamma=\sigma^{2}
$$

where

$$
\varphi_{e s}(\gamma)=\left[\varphi_{q}(\gamma)\right]^{q} / \int\left[\varphi_{q}(\gamma)\right]^{q} d \gamma
$$


represents the escort probability distribution $[14,12]$. The Lagrangian function is given as

$L\left(\varphi_{q}, \gamma\right)=\left(\begin{array}{l}\frac{1-\int_{-\infty}^{+\infty}\left[\varphi_{q}(\gamma)\right]^{q} d \gamma}{q-1}+\lambda_{1}\left(1-\int_{-\infty}^{\infty} \varphi_{q}(\gamma) d \gamma\right) \\ +\lambda_{2}\left(\mu-\int_{-\infty}^{\infty} \gamma \varphi_{e s}(\gamma) d \gamma\right)+\lambda_{3}\left(\sigma^{2}-\int_{-\infty}^{\infty}(\gamma-\mu)^{2} \varphi_{e s}(\gamma) d \gamma\right)\end{array}\right)$,

where Lagrangian multipliers are expressed as $\lambda_{1}, \lambda_{2}$ and $\lambda_{3}$. Employing EulerLagrange equation:

$$
\frac{\partial L}{\partial \varphi_{q}}-\frac{d}{d \gamma}\left(\frac{\partial L}{\partial \varphi_{q}^{\prime}}\right)=0
$$

The $q$-Lognormal PDF incorporating non-extensive Tsallis' parameter ' $q$ ' for long term fading channels with received $\operatorname{SNR}(\gamma)$ is given from Eq. 8 together with Eq. 1 to Eq. 7 as [23]

$$
\varphi_{q}(\gamma)=\frac{1}{\xi \gamma}\left(1+\alpha\left(\frac{\ln (\gamma)-\mu}{\sigma}\right)^{2}\right)^{\frac{1}{1-q}}, \gamma>0
$$

where $\alpha=\left(\frac{q-1}{3-q}\right)$ and the normalized constant $\xi$ is defined as

$$
\xi=\sigma \frac{\Gamma\left(\frac{3-q}{2 q-2}\right)}{\Gamma\left(\frac{1}{q-1}\right)} \sqrt{\frac{\pi}{\alpha}}, \quad 1<q<3 .
$$

When $q \rightarrow 1$, the above Eq. 9 portrays the conventional log-normal model given as

$$
\varphi_{\log }(\gamma)=\frac{1}{\sqrt{2 \pi} \sigma \gamma} e^{-\frac{(\ln (\gamma)-\mu)^{2}}{2 \sigma^{2}}}, \gamma>0
$$

where $\sigma$ and $\mu$ represents standard deviation and mean of $\ln (\gamma)$.

In Fig. 1 it is perceived that when $q>1$, the notion of long-tailed distribution is achieved which is important in characterizing the extreme fluctuations in the outliers.

It is well-known that the lognormal distribution characterizes long term fading or shadowing effects [1] . However, the proposed model well characterizes the tail variations over the synthesized fading signals [23]. From Fig.2, it is noted that the log-normal model does not provide better agreement with the synthesized synthetic fading signals. To mimic real-time fading scenarios, the fading signals are produced with the help of MATLAB tool. It is observed that the proposed $q$-Lognormal distribution based on Tsallis' entropy widely captures the extreme fluctuations in the tail regions of the fading signals. In this setting, the presented model signified an excellent agreement to the outliers of the synthesized fading signals in the continuous range, $1<q<3$. The presenetd $q$-Lognormal distribution well captured the synthetic fading signals with respect to $q=2.56$ as shown in Fig.3 [23]. 


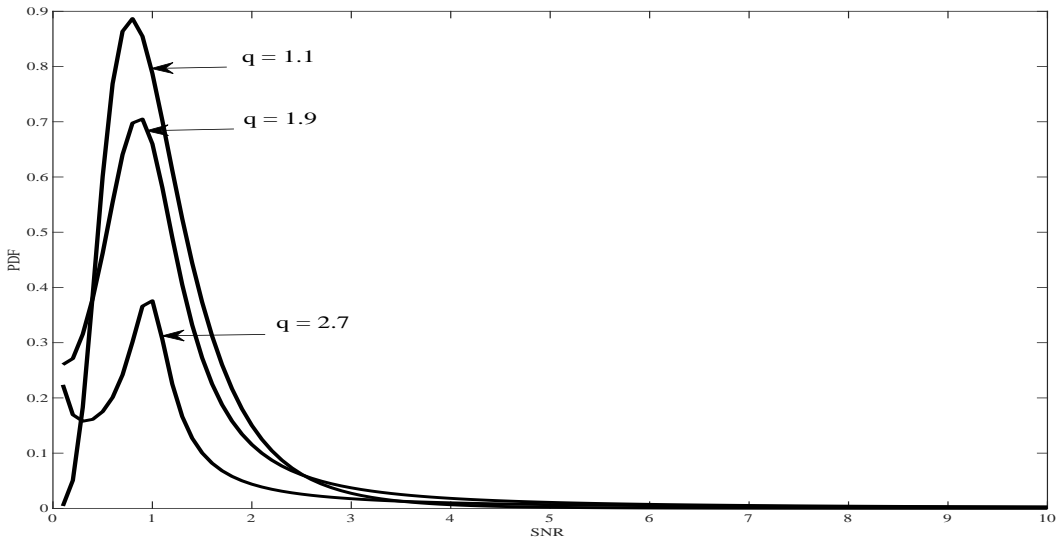

Fig. 1 Illustration of $q$-Lognormal model of SNR $(\gamma)$ with respect to different values of parameter $q$.

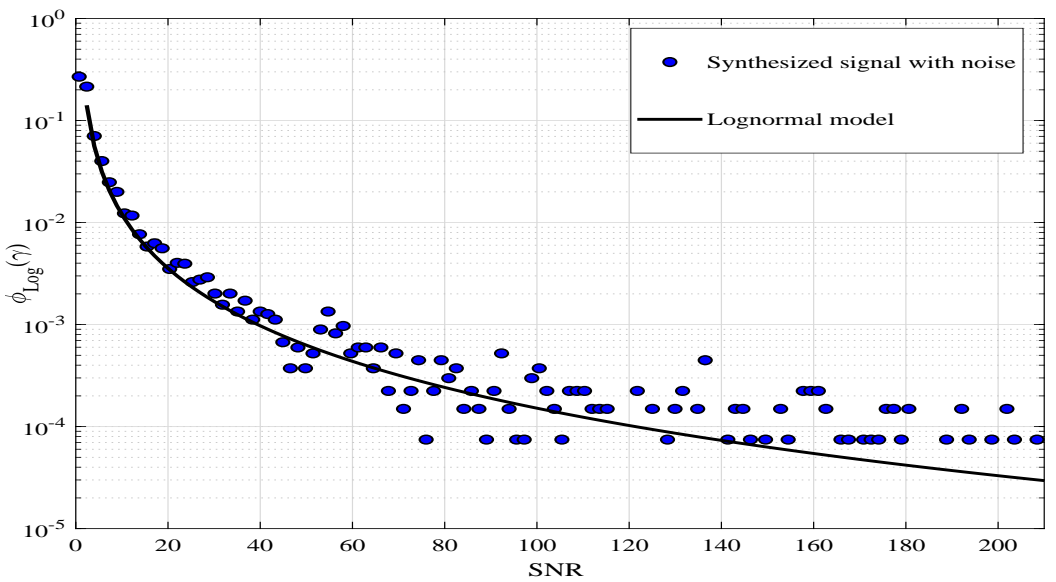

Fig. 2 Illustration of log-normal model corresponding to synthesized signal.

\section{Goodness of fit and Relative error}

\subsection{Goodness of fit}

The goodness of fit of $q$-Lognormal distribution in reference to synthesized fading signal is portrayed [9]. The null hypothesis $H_{0}$ : the generated fading data is in agreement with the theoretical $q$-Lognormal distribution is validated by employing $\chi^{2}$-test. The value of degrees of freedom (d.f) $=\mathrm{n}-1-1$, where $\mathrm{n}=19$ is the number of bins in the histogram [9] of the synthetic signal. Since, JS measure is used to estimate the parameter $q$, there is an unit decrement in the 


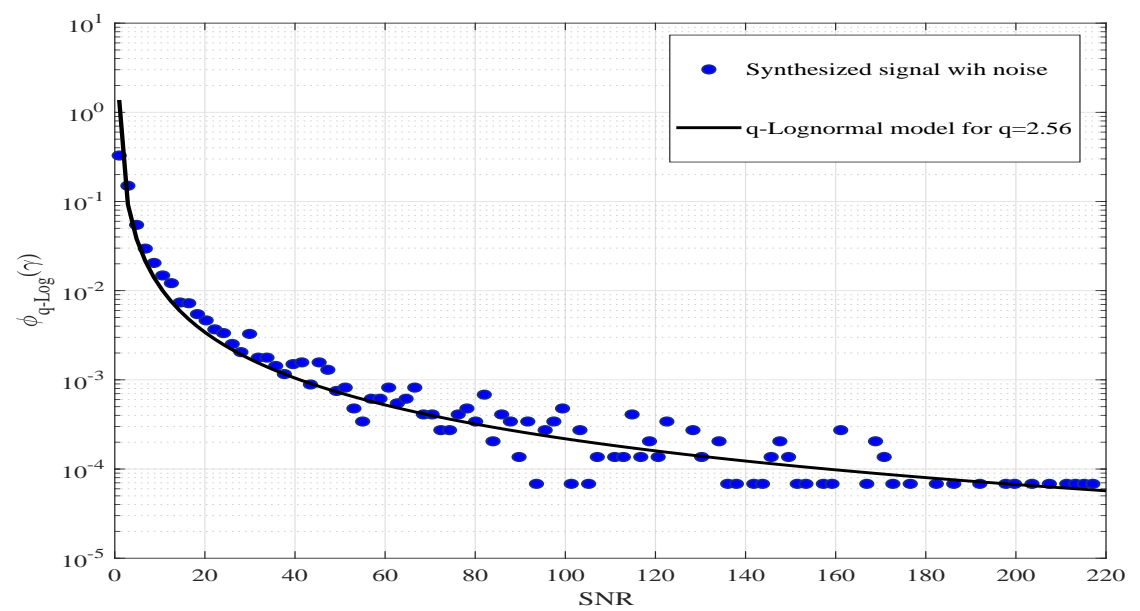

Fig. 3 Illustration of $q$-Lognormal model with respect to synthesized signal for $q=2.56$.

d.f. Furthermore, one d.f is also reduced by the normalization condition. The calculated $\chi^{2}(\mathrm{obs})=1.39$ is less than the tabulated value of $\chi_{19}^{2}(0.05)=44.181$. Hence, the proposed null hypothesis is accepted i.e., the stated model well characterizes the wide range of wireless fading scenarios.

Table 1 List of used Mathematical symbols and notations along with their descriptions

\begin{tabular}{|c|c|}
\hline Symbols and Functions & Description \\
\hline$\Gamma$ & Random variable corresponding to received average SNR \\
\hline$T_{q}()$. & Tsallis entropy \\
\hline$\varphi_{e s}()$. & Escort probability distribution \\
\hline$\mu$ and $\sigma$ & Mean and Standard deviation \\
\hline $\mathrm{L}()$. & Lagrangian function \\
\hline$\lambda_{1}, \lambda_{2}$ and $\lambda_{3}$ & Lagrangian multipliers \\
\hline$\varphi_{q}()$. & -Lognormal PDF \\
\hline$q$ & Tsallis non-extensive parameter \\
\hline$\xi$ & Normalization constant \\
\hline $\mathrm{KL}()$. & Kullback-Liebler divergence \\
\hline $\mathrm{JS}()$. & Jensen-Shannon divergence \\
\hline $\mathrm{C}$ & Instantaneous channel capacity \\
\hline $\mathrm{F}()$. & CDF of channel capacity \\
\hline$F_{1}[\mathrm{a}, \mathrm{b}, \mathrm{c} ; \mathrm{z}]$ & Gauss Hypergeometric function \\
\hline$\Gamma()$. & Gamma function \\
\hline$\Phi()$. & Standard normal variate \\
\hline &
\end{tabular}




\subsection{Relative error}

The presented $q$-Lognormal model in Eq. 1 is compared against the well known lognormal distribution in terms of relative error. The Fig.4 illustrates that the proposed distribution has lower relative error in contrary to the conventional lognormal model towards defining the shadowing phenomena.

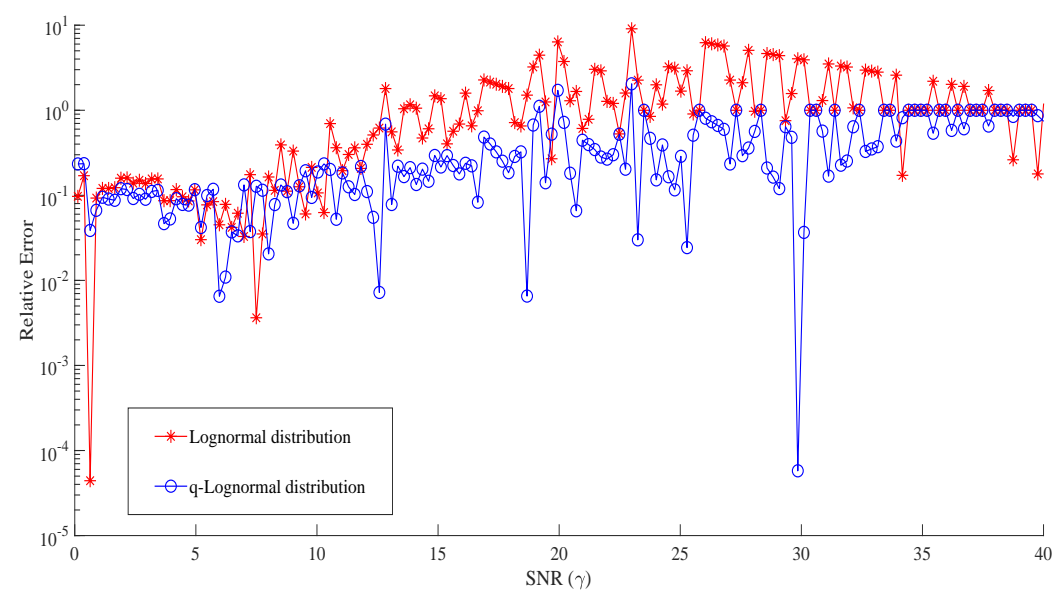

Fig. 4 Comparison of Relative error of $q$-Lognormal model in contrast to conventional Lognormal distribution.

\section{Generic Symmetric JS estimation}

The selection of an appropriate model is a challenge while characterizing various fading channels because significant prior information about the class of exact distributions is obscure $[9,26]$. The proposed $q$-Lognormal distribution is equipped with the real valued Tsallis' parameter ' $q$ ', that can be evaluated from the observed histogram of the synthesized fading data. In this context, the generic Kullback-Leibler (KL) metric provides the relative distance amongst the proposed model and the referenced synthetic distribution viz., the synthesized fading data. As KL measure is asymmetric with respect to the synthetic fading data and the measure is not bounded, the calculated value of the non-extensive parameter ' $q$ ' may lead to erroneous characterization of the fading data. In this context, the estimated value of parameter ' $q$ ' is within the vicinity of the minimal value of symmetric JS measure. In Fig.4 and Fig.5 it is observed that the value of real-valued Tsallis' parameter ' $q$ ' is estimated and the measure is bounded i.e., the symmetric JS measure value is confined within the range 0 to 1 . 
The generic KL measure between the proposed model $p_{q l}(u, v)$ and referenced synthetic normalized histogram $q(u, v)$ in Tsallis' context is expressed as

$$
\begin{aligned}
K L\left(p_{q l}(u, v) \| q(u, v)\right) & =\frac{1}{q-1} \int\left[p_{q l}(u, v)\right]^{q}\left(1-\left(\frac{q(u, v)}{p_{q l}(u, v)}\right)^{q-1}\right) d u \\
& -\int\left(\frac{p_{q l}(r, t)}{q(u, v)}-1\right)[g(u, v)]^{q} d u
\end{aligned}
$$

Discretizing the above equation yields

$$
\begin{aligned}
K L\left(p_{q l}(u, v) \| q(u, v)\right) & =\frac{1}{q-1} \sum_{u}\left[p_{q l}(u, v)\right]^{q}\left(1-\left(\frac{q(u, v)}{p_{q l}(u, v)}\right)^{q-1}\right) \Delta u \\
& -\sum_{u}\left(\left(\frac{p_{q l}(u, v)}{q(u, v)}\right)-1\right)[q(u, v)]^{q} \Delta u
\end{aligned}
$$

A generalized symmetric measure proposed by Jensen-Shannon is given as

$$
\begin{aligned}
J S\left(p_{q l}(u, v) \| q(u, v)\right) & =\frac{1}{2} K L\left(p_{q l}(u, v) \| \frac{p_{q l}(u, v)+q(u, v)}{2}\right) \\
& +\frac{1}{2} K L\left(q(u, v) \| \frac{p_{q l}(u, v)+q(u, v)}{2}\right)
\end{aligned}
$$

The different values of the parameter $q$ corresponding to the synthesized fading data have been computed using the following designed algorithm.

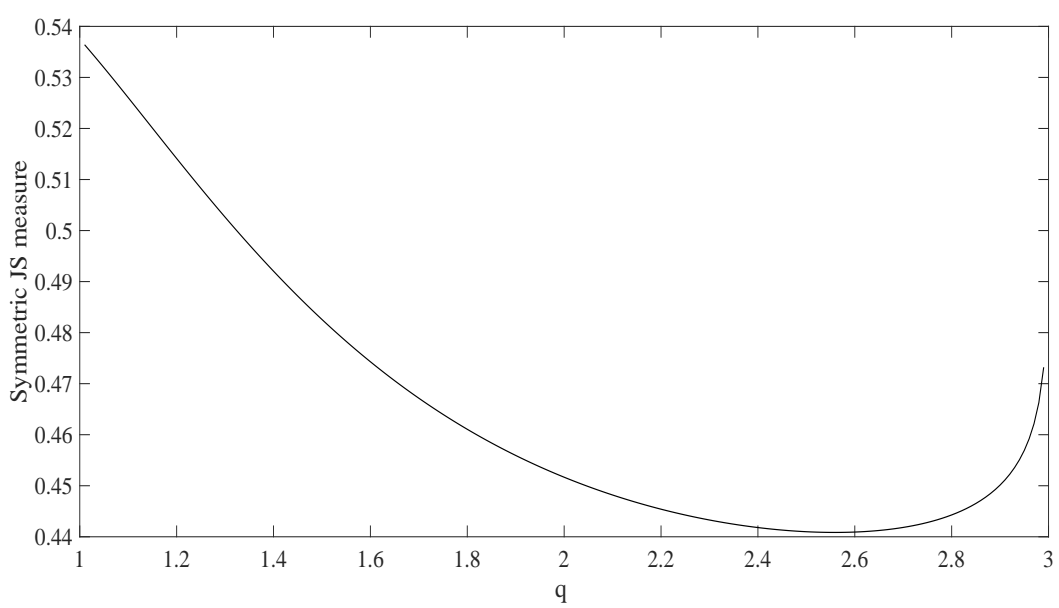

Fig. 5 Symmetric JS measure for $q$-Lognormal model and synthetic signal of $O\left(10^{8}\right)$ corresponding to $q$. 

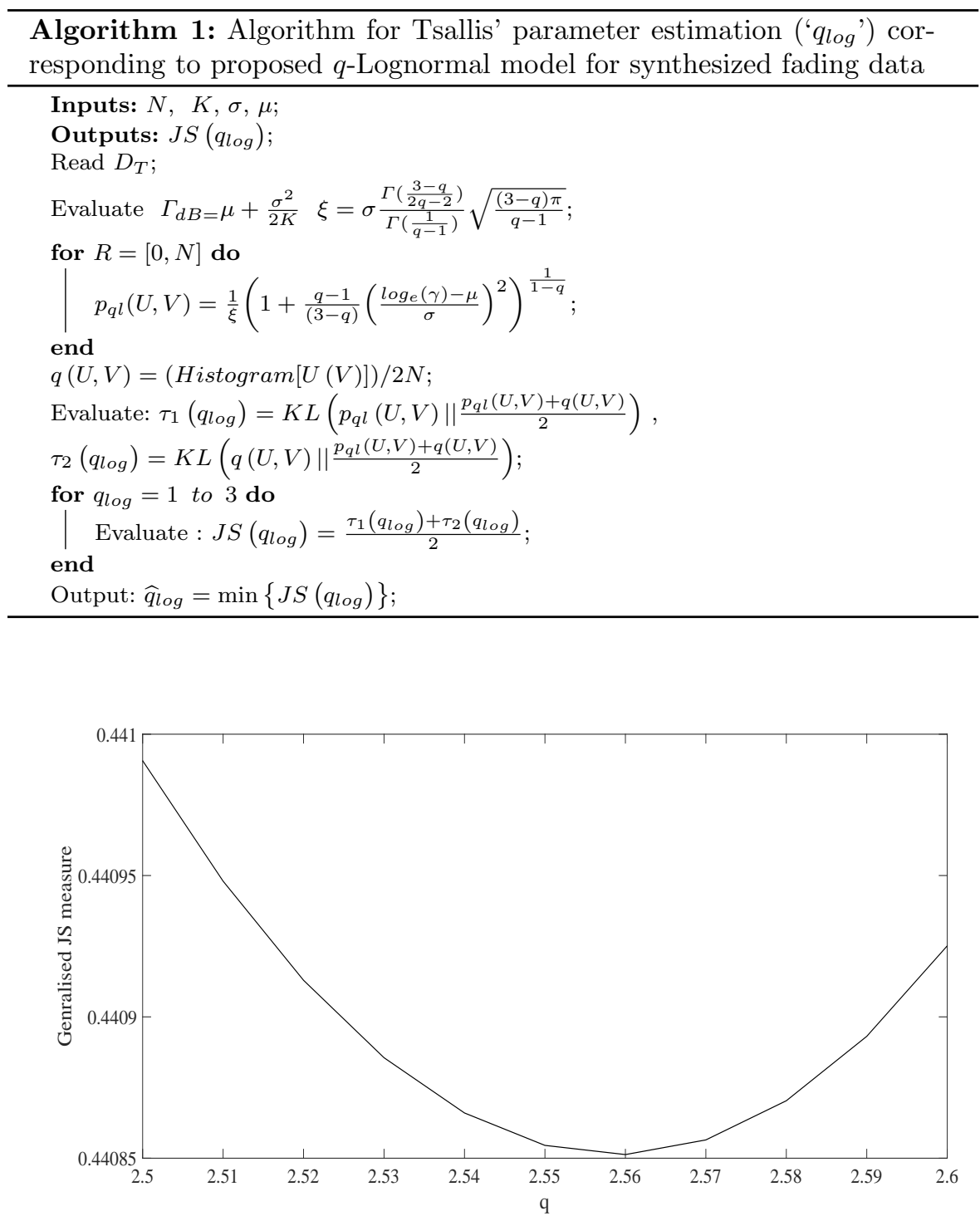

Fig. 6 Cropping of Fig.5 in the close neighborhood of $q=2.5$.

The Fig. 5 highlights the estimated value of $q$ in the vicinity of 2.5 and it is observed that the exact value of $q$ for the generated synthetic signal is 2.56 . The same synthetic data has been used in Fig.3 along the with proposed $q$ Lognormal model and it is found to be in tight agreement with the generated data for $q=2.56$. For more clarity, Table 1 provides the tabulated value of JS divergence for various range of ' $q$ ' in the vicinity of 2.5 . From Table 1, the optimal value for JS measure is obtained at ' $q$ ' $=2.56$ i.e., $J S_{q L o g}=0.4451012$. 
Table 2 Tabulated value of JS divergence of the synthesized fading signal in tte vicinity of $q=2.5$.

\begin{tabular}{|c|c|c|c|c|c|}
\hline$q$ & JSvalue & $q$ & JSvalue & $q$ & JSvalue \\
\hline 2.49 & 0.4454482 & 2.50 & 0.4453819 & 2.51 & 0.4453371 \\
\hline 2.52 & 0.4452185 & 2.53 & 0.4452169 & 2.54 & 0.4452172 \\
\hline 2.55 & 0.4451129 & $\mathbf{2 . 5 6}$ & $\mathbf{0 . 4 4 5 1 0 1 2}$ & 2.57 & 0.4451018 \\
\hline 2.58 & 0.4451104 & 2.59 & 0.4452216 & 2.60 & 0.4453124 \\
\hline
\end{tabular}

\section{Analytical Model for Channel Capacity over $q$-Lognormal distribution}

The channel capacity is stated as the probability of achieving a small error rate while maintaining the maximum transmission capacity of the channel [19]. The instantaneous channel capacity is obtained as [13]

$$
C=\log (1+\gamma)
$$

Here $\gamma$ represents the $q$-Lognormal random variable with PDF defined as

$$
f(\gamma)=\frac{\vartheta}{\xi \gamma}\left(1-\frac{1-q}{(3-q) \sigma^{2}}(\vartheta \ln (\gamma)-\mu)^{2}\right)^{\frac{1}{1-q}}, \gamma>0, \sigma>0 .
$$

where $\xi$ denotes the normalization constraint expressed as

$$
\xi=\sigma \sqrt{\frac{(3-q) \pi}{(q-1)}} \frac{\Gamma\left(\frac{3-q}{2 q-2}\right)}{\Gamma\left(\frac{1}{q-1}\right)}, \quad 1<q<3 .
$$

where $\sigma$ and $\mu$ represents the standard deviation and mean for the random variable $\log (\gamma)$ which is expressed in decibels $(\mathrm{dB})$ and $\vartheta=10 / \ln (10)$. From Eq. 15 and Eq. 16, the PDF of channel capacity is expressed in closed form as

$$
f(c)=\frac{\vartheta}{\xi\left(1-e^{-c}\right)}\left(1-\frac{1-q}{(3-q) \sigma^{2}}\left(\vartheta \log _{e}(\exp (c)-1)-\mu\right)^{2}\right)^{\frac{1}{1-q}}, c>0 .
$$

The mathematical expression representing the CDF of channel capacity over the proposed $q$-Lognormal model is obtained as

$$
F(c)=\int_{-\infty}^{c} \frac{\vartheta}{\xi\left(1-e^{-c}\right)}\left(1-\frac{1-q}{3-q}\left(\frac{\vartheta \ln \left(e^{c}-1\right)-\mu}{\sigma}\right)^{2}\right)^{\frac{1}{1-q}} d c .
$$

Substituting $\vartheta \ln \left(e^{c}-1\right)=x$ and after some mathematical operations we obtain:

$$
F(c)=\frac{1}{\xi} \int_{-\infty}^{\vartheta \ln \left(e^{c}-1\right)-\mu}\left(1-\frac{1-q}{3-q}\left(\frac{x-\mu}{\sigma}\right)^{2}\right)^{\frac{1}{1-q}} d x
$$




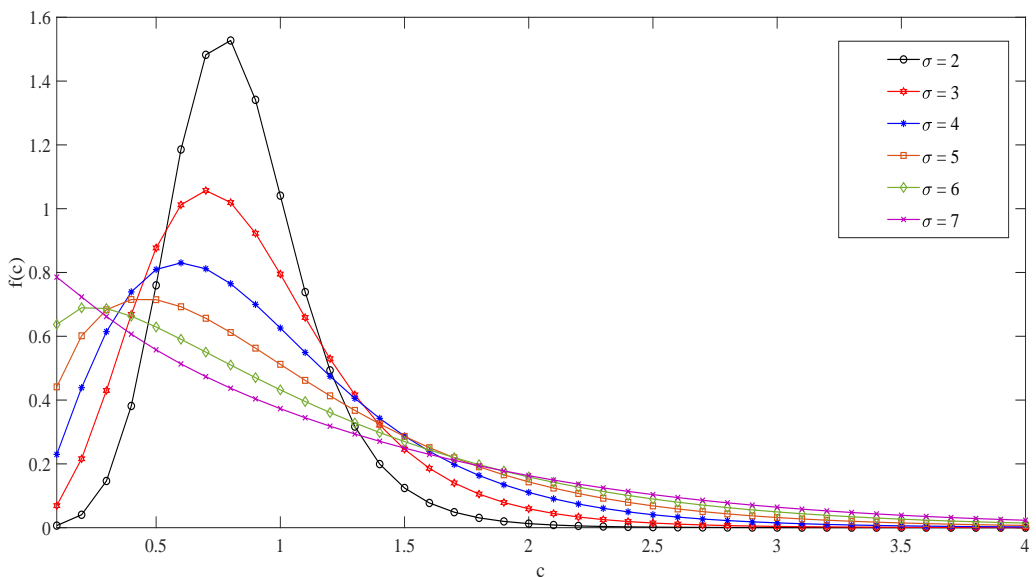

Fig. 7 PDF of $q$-Lognormal channel capacity over $q=1.2, \mu_{d B}=1$ with different range of $\sigma_{d B}$.

Replacing $\frac{x-\mu}{\sigma}=y$ we obtain

$$
F(c)=\frac{\sigma}{\xi} \int_{-\infty}^{\frac{\vartheta \ln \left(e^{c}-1\right)-\mu}{\sigma}} \frac{\vartheta}{\left(1-e^{-c}\right)}\left(1+\frac{q-1}{3-q}(y)^{2}\right)^{\frac{1}{1-q}} d y
$$

Finally, the CDF of channel capacity corresponding to $q$-Lognormal model is obtained in closed form as

$$
F(c)=\frac{1}{2}+\frac{\vartheta \ln \left(e^{c}-1\right)-\mu}{\sigma \xi}{ }_{2} F_{1}\left[\frac{1}{2}, \frac{1}{q-1}, \frac{3}{2}, \frac{1-q}{3-q}\left(\frac{\vartheta \ln \left(e^{c}-1\right)-\mu}{\sigma}\right)^{2}\right] .
$$

where ${ }_{2} F_{1}$ is the Gauss Hypergeometric function and is defined as [6]

$$
{ }_{2} F_{1}[\mathrm{a}, \mathrm{b}, \mathrm{c} ; \mathrm{z}]=\frac{\Gamma(c)}{\Gamma(a) \Gamma(b)} \sum_{n=0}^{\infty} \frac{\Gamma(a+n) \Gamma(b+n)}{\Gamma(c+n)} \frac{z^{n}}{n !} .
$$

and $\Gamma($.$) denotes the gamma function defined as$

$$
\Gamma(v)=\int_{0}^{\infty} w^{v-1} e^{-w} d w
$$

From Eq. 18 and Eq. 22, PDF and CDF graphs are plotted correponding to $q=1.2$ and different range of $\sigma_{d B}$ for realistic $\mathrm{dB}$ spread as portrayed in Fig.7 and Fig.8. 


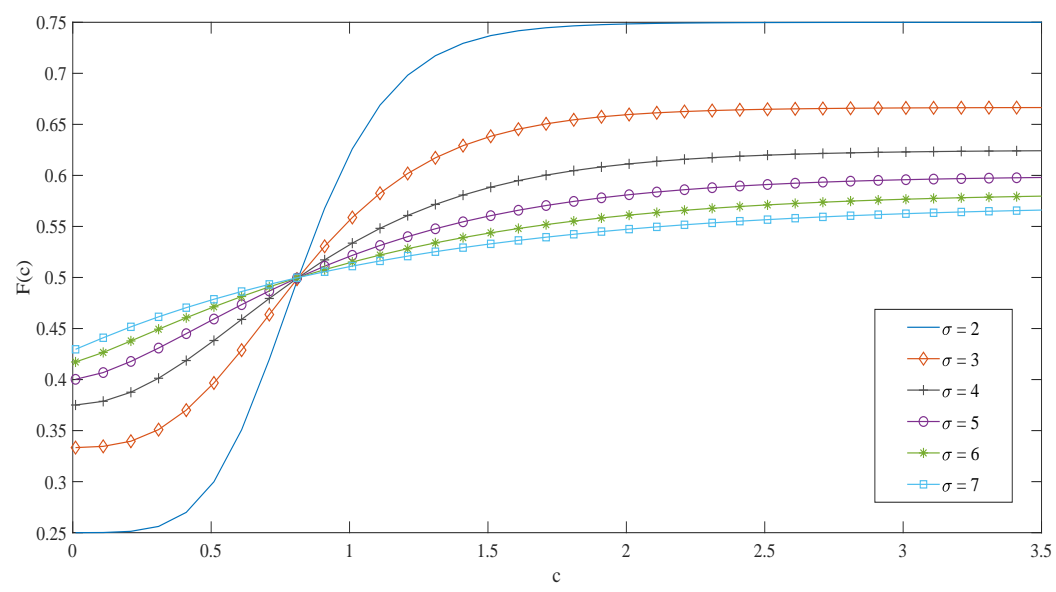

Fig. $8 \mathrm{CDF}$ of $q$-Lognormal channel capacity over $q=1.2, \mu_{d B}=1$ with different range of $\sigma_{d B}$.

\section{$6 K^{\text {th }}$ order $q$-Lognormal Moments corresponding to Channel Capacity in Shadowing Environment}

It is worth noting that the computaion of higher order moments is essential for analyzing important statistical behavior of channel capacity. In this context, this section derives the analytical expressions of a few higher moments of the instantaneous channel capacity. Laourine et.al [20] have computed the accurate expressions in terms of convergent infinite series portraying the higher order moments of channel capacity over lognormal fading scenarios. In context of maintaining completeness, we provide only the first and second order moments corresponding to channel capacity as derived in [20]. The first order moment is mathematically expressed as

$$
E[C]=\int_{0}^{\infty} \frac{\vartheta c}{\left(1-e^{-c}\right) \sqrt{2 \pi \sigma^{2}}} e^{\left(-\left(\frac{\vartheta \log _{e}(\exp (c)-1)-\mu}{\sigma \sqrt{2}}\right)^{2}\right)} d c .
$$

From this Equation the analytical expression for first order moment is obtained due to Laourine et.al.[20] as

$E[C]=\left\{\begin{array}{l}\sum_{\vartheta=1}^{\infty} \frac{(-1)^{\vartheta+1}}{\vartheta} \operatorname{erfcx}\left(\frac{\vartheta \sigma}{\sqrt{2}}-\frac{\mu}{\sqrt{2} \sigma}\right)+\sum_{\vartheta=1}^{\infty} \frac{(-1)^{\vartheta+1}}{\vartheta} \operatorname{erfcx}\left(\frac{\vartheta \sigma}{\sqrt{2}}+\frac{\mu}{\sqrt{2} \sigma}\right)+ \\ (\mu) \operatorname{erfcx}\left(-\frac{\mu}{\sqrt{2} \sigma}\right)+\sigma \sqrt{\frac{2}{\pi}}\end{array}\right\} \frac{e^{-\frac{\mu^{2}}{2 \sigma^{2}}}}{2}$.

The expression for second order moment is given as,

$$
E\left[C^{2}\right]=\int_{0}^{\infty} \frac{\vartheta c^{2}}{\left(1-e^{-c}\right) \sqrt{2 \pi \sigma^{2}}} e^{\left(-\left(\frac{\vartheta \log _{e}(\exp p(c)-1)-\mu}{\sigma \sqrt{2}}\right)^{2}\right)} d c .
$$


From this Equation the analytical expression for second order moment is obtained due to Laourine et.al.[20] as:

$$
E\left[C^{2}\right]=\left\{\begin{array}{l}
\sum_{\vartheta=1}^{\infty} 2 \frac{(-1)^{\vartheta+1}}{\vartheta}\left(\operatorname{erfcx}\left(\frac{\vartheta \sigma}{\sqrt{2}}-\frac{\mu}{\sqrt{2} \sigma}\right)\left(\mu-\vartheta \sigma^{2}\right)+\sigma \sqrt{\frac{2}{\pi}}\right)+ \\
\sum_{\vartheta=1}^{\infty} 2 \frac{(-1)^{\vartheta}}{\vartheta} \operatorname{erfcx}\left(\frac{\vartheta \sigma}{\sqrt{2}}-\frac{\mu}{\sqrt{2} \sigma}\right)(\psi(\vartheta)-\psi(1))+ \\
\sum_{\vartheta=1}^{\infty} 2 \frac{(-1)^{\vartheta}}{\vartheta} \operatorname{erfcx}\left(\frac{\vartheta \sigma}{\sqrt{2}}+\frac{\mu}{\sqrt{2} \sigma}\right)(\psi(\vartheta)-\psi(1))+ \\
\operatorname{erfcx}\left(-\frac{\mu}{\sqrt{2} \sigma}\right)\left(\mu^{2}+\sigma^{2}\right)+\sigma \mu \sqrt{\frac{2}{\pi}}
\end{array}\right\} \frac{e^{-\frac{\mu^{2}}{2 \sigma^{2}}}}{2}
$$

However, we need to compute the higher order moments of channel capacity corresponding to the proposed $q$-Lognormal model. Since the proposed model is not analytically tractable we perform rigorous Monte-Carlo simulation schemes averaging over $O\left(10^{7}\right)$ simulation paths. Fig.9 and Fig.10 represents the plot of the first and second order moments corresponding to the channel capacity i.e. $E[C]$ and $E\left[C^{2}\right]$ over the proposed model for $q=1.2$. The obtained results have been validated using the Symbolic Math Toolbox of MATLAB and is observed that the simulation results are in close agreement with the results obtained from symbolic integration. Moreover, for $q \rightarrow 1$, the proposed model mimics the conventional log-normal distribution for characterizing the shadowing phenomena $[23,3]$. In this context, we obtain the closed form expressions of the moments for channel capacity using Holtzman approximation [11].

The first moment corresponding to channel capacity over the proposed $q$ Lognormal model is expressed as

$$
E[C]_{q L o g}=\int_{0}^{\infty} c \frac{\vartheta}{\xi\left(1-e^{-c}\right)}\left(1+\frac{q-1}{3-q}\left(\frac{\vartheta \log _{e}(\exp (c)-1)-\mu}{\sigma}\right)^{2}\right)^{\frac{1}{1-q}} d c
$$

The second moment corresponding to channel capacity over the proposed $q$ Lognormal model is expressed as

$$
E\left[C^{2}\right]_{q L o g}=\int_{0}^{\infty} c^{2} \frac{\vartheta}{\xi\left(1-e^{-c}\right)}\left(1+\frac{q-1}{3-q}\left(\frac{\vartheta \log _{e}(\exp (c)-1)-\mu}{\sigma}\right)^{2}\right)^{\frac{1}{1-q}} d c .
$$

When $q \rightarrow 1$ Eq. 29 reduces to the first order moment of channel capacity corresponding to log-normal distribution and is expressed as

$$
E[C]_{\log }=\int_{0}^{\infty} c f_{\log }(c) d c=\int_{0}^{\infty} c \frac{\vartheta}{\left(1-e^{-c}\right) \sqrt{2 \pi \sigma^{2}}} \mathrm{e}^{\left(-\frac{1}{2}\left(\frac{\vartheta \log _{e}(\exp p(c)-1)-\mu}{\sigma}\right)^{2}\right)} d c .
$$

The closed form expression of the integral in Eq. 31 is obtained by employing the method defined by Holtzman [11]. The PDF incorporating the standard 


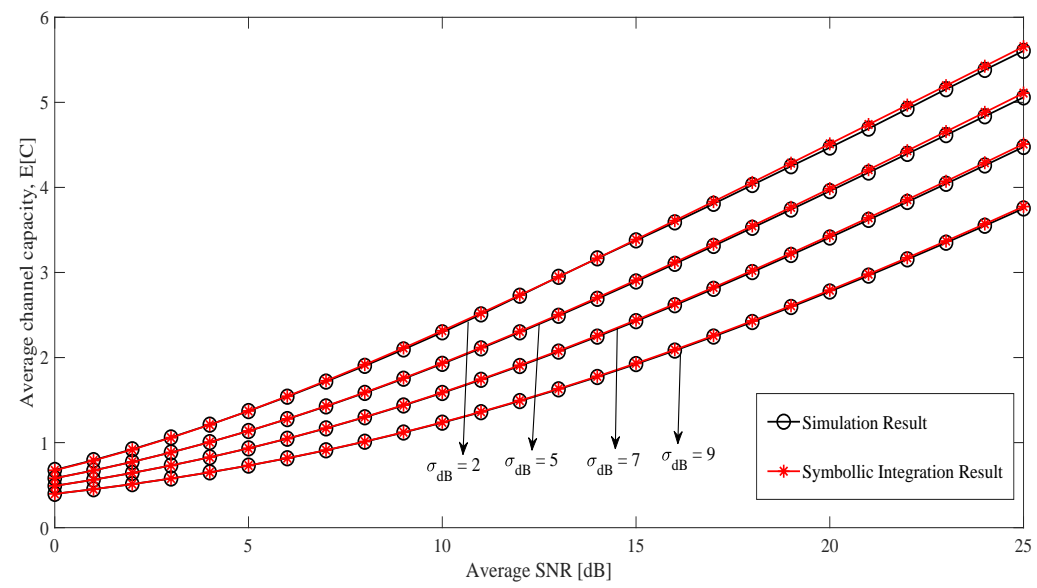

Fig. 9 Plot of first moment of Average channel capacity (E[C]) over $q$-Lognormal model $(q=1.2)$ corresponding to different $\sigma_{d B}$.

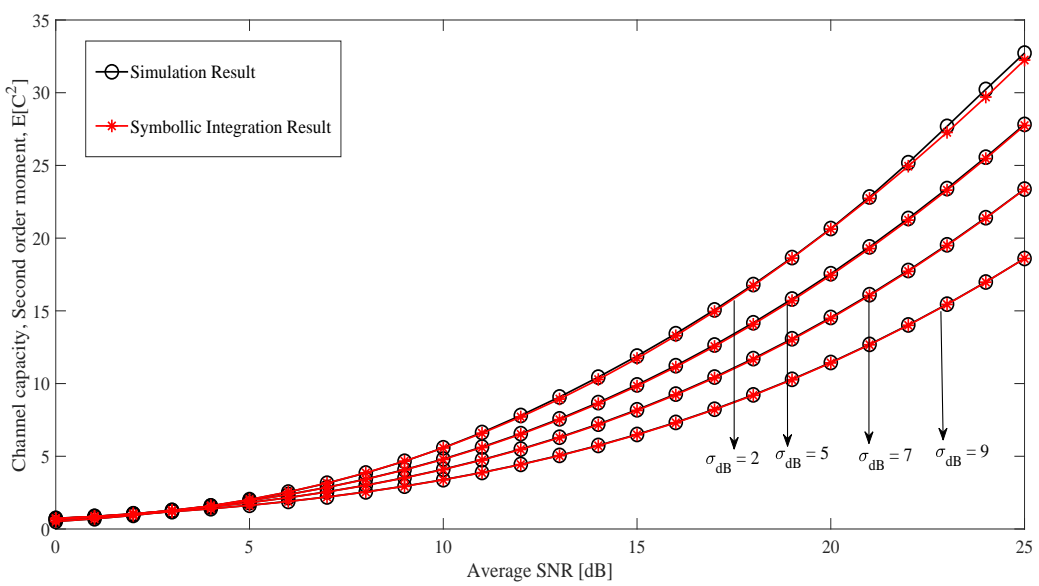

Fig. 10 Plot of second moment of channel capacity $\left(E\left[C^{2}\right]\right)$ over $q$-Lognormal model $(q=1.2)$ corresponding to different $\sigma_{d B}$.

normal distribution, with random variable $\mathrm{B} \sim \mathrm{N}\left(\mu, \sigma^{2}\right)$ can be portrayed as three points at $\mu, \mu+\sqrt{3} \sigma, \mu-\sqrt{3} \sigma$. The normal variate $\Phi(\beta)$ is obtained as

$$
\Phi(\beta)=\frac{1}{6} \delta(\beta-(\mu+\sqrt{3} \sigma))+\frac{2}{3} \delta(\beta-\mu)+\frac{1}{6} \delta(\beta-(\mu+\sqrt{3} \sigma)) .
$$

Let $\zeta$ be a function with random variable $B$ having variance $\sigma^{2}$ and mean $\mu$. Performing Taylor's series expansion it is obtained

$$
\zeta(\beta)=\zeta(\mu)+(\beta-\mu) \zeta^{\prime}(\mu)+\frac{1}{2}(\beta-\mu)^{2} \zeta^{\prime \prime}(\mu)+\ldots
$$


By performing mathematical expectation, Holtzman [11] resulted in the approximation given as

$$
E[\zeta(\beta)] \approx \zeta(\mu)+\frac{1}{2} \zeta^{\prime \prime}(\mu) \sigma^{2} .
$$

Employing Stirling's formula for evaluating expansion in differences and taking expectation, $E[\zeta(\beta)]$ is obtained as [11]

$$
E[\zeta(\beta)] \approx \zeta(\mu)+\frac{\sigma^{2}}{2} \frac{\zeta(\mu-h)-2 \zeta(\mu)+\zeta(\mu+h)}{h^{2}} .
$$

where $h$ denotes the difference parameter and is appropriated as $h=\sigma \sqrt{3}$, hence Eq. 35 evaluates to

$$
E[\zeta(\beta)] \approx \frac{1}{6} \zeta(\mu+\sqrt{3} \sigma)+\frac{2}{3} \zeta(\mu)+\frac{1}{6} \zeta(\mu-\sqrt{3} \sigma) .
$$

Now, $k^{\text {th }}$ order moment of the channel capacity is defined as

$$
E\left[C^{k}\right]_{\log }=E\left[(\log (1+\gamma))^{k}\right]_{\log }=\left(\int_{\gamma=0}^{\infty} \frac{1}{\sigma \gamma \sqrt{2 \pi}} \exp \left(-\frac{1}{2}\left(\frac{\log _{e}(\gamma)-\mu}{\sigma}\right)^{2}\right)\right) .
$$

Substituting $\log _{e}(\gamma)=\beta$, so, $\gamma=\exp (\beta)$, the Eq. 37 is transformed as

$$
E\left[C^{k}\right]_{\log }=\int_{\beta=-\infty}^{\infty} \frac{1}{\sigma \sqrt{2 \pi}} \exp \left(-\frac{1}{2}\left(\frac{\beta-\mu}{\sigma}\right)^{2}\right)(\log (1+\exp (\beta)))^{k} d \beta .
$$

From Eq. 36 and Eq. 38, an accurate analytical expression for $k_{t h}$ moment corresponding to the channel capacity is obtained as

$$
E\left[C^{k}\right]_{\log }=\frac{1}{6} \zeta(\mu+\sqrt{3} \sigma)+\frac{2}{3} \zeta(\mu)+\frac{1}{6} \zeta(\mu-\sqrt{3} \sigma) .
$$

where $\zeta(\beta)=(\log (1+\exp (\beta)))^{k}$. Substituting different values for $k$, we obtain $E[C]=\frac{1}{6} \log (1+\exp (\mu+\sqrt{3} \sigma))+\frac{2}{3} \log (1+\exp (\mu))+\frac{1}{6} \log (1+\exp (\mu-\sqrt{3} \sigma))$.
$E\left[C^{2}\right]=\frac{1}{6}(\log (1+\exp (\mu+\sqrt{3} \sigma)))^{2}+\frac{2}{3}(\log (1+\exp (\mu)))^{2}+\frac{1}{6}(\log (1+\exp (\mu-\sqrt{3} \sigma)))^{2}$.
$E\left[C^{3}\right]=\frac{1}{6}(\log (1+\exp (\mu+\sqrt{3} \sigma)))^{3}+\frac{2}{3}(\log (1+\exp (\mu)))^{3}+\frac{1}{6}(\log (1+\exp (\mu-\sqrt{3} \sigma)))^{3}$.
$E\left[C^{4}\right]=\frac{1}{6}(\log (1+\exp (\mu+\sqrt{3} \sigma)))^{4}+\frac{2}{3}(\log (1+\exp (\mu)))^{4}+\frac{1}{6}(\log (1+\exp (\mu-\sqrt{3} \sigma)))^{4}$.

In Fig.11, the plot of analytical expression for first moment of channel capacity corresponding to log-normal model (Eq. 40) has been presented. Moreover, the 


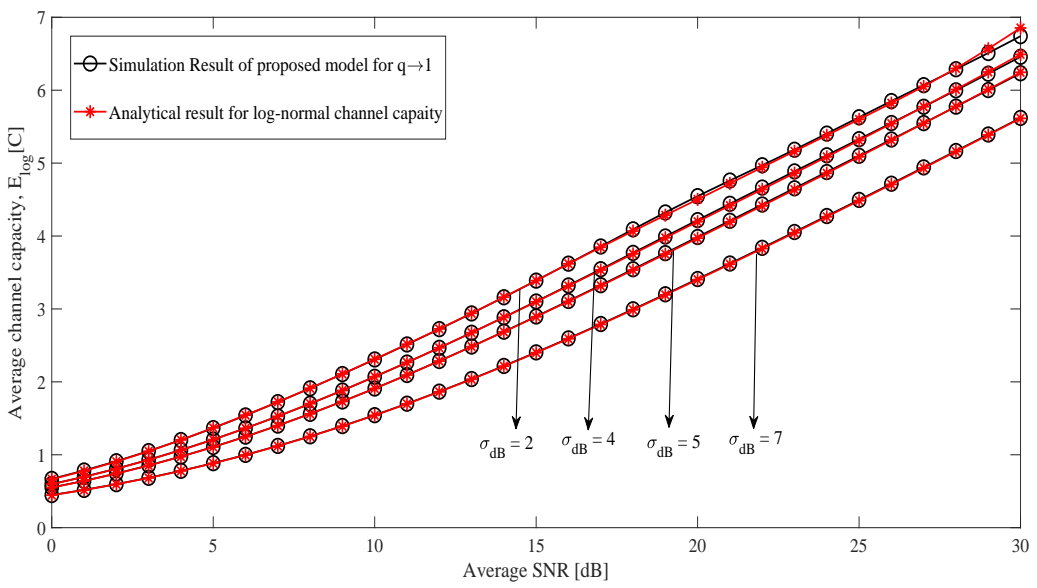

Fig. 11 Plot of first moment of log-normal and $q$-Lognomal $(q \rightarrow 1)$ channel capacity vs Average SNR corresponding to different range of $\sigma_{d B}$.

first order moment portraying $q$-Lognormal model for $q \rightarrow 1$ i.e., mimicking the log-normal distribution has also been plotted by performing rigorous MonteCarlo simulation schemes. It can be observed that both the results are in good agreement with each other which proves the validity and accuracy of the simulation schemes employed.

\section{Computation of Coefficient of Variation (CV)}

The statistical measure, coefficient of variation $(\mathrm{CV})$, is an important metric for the channel capacity over the proposed $q$-Lognormal model. It represents the dispersion of the signal points over the wireless fading signals around the mean. It is expressed as the relative change in the square root of variance with respect to its mean, and is an important measure for estimating the fluctuation rate in channel capacity of the fading signals [29]. It is obtained as,

$$
C V=\frac{1}{E[C]} \sqrt{\operatorname{Var}(C)}
$$

The plot of CV against average SNR over $q$-Lognormal channel capacity is presnted in Fig.12, and it signifies that the coefficient of variation portrays higher values corresponding to lower arguments of received average SNR. The fluctuation rate in the channel capacity drops with an increase in the average SNR. It is noted that the simulation results and the symbolic integration results are in closed agreement with each other. The symbolic integration has been performed using Symbolic Math Toolbox in MATLAB. 


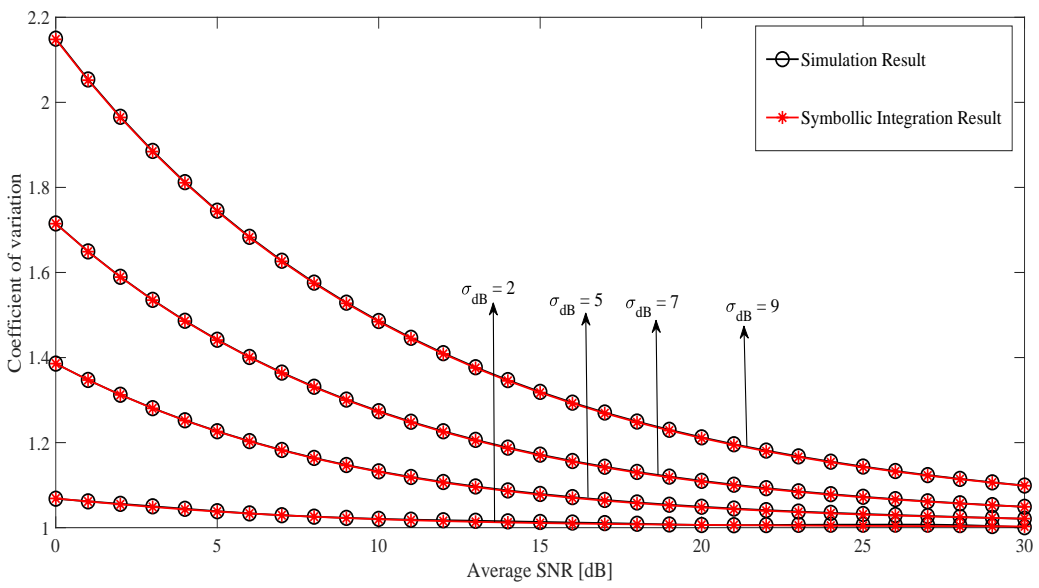

Fig. 12 Plot of CV for $q$-Lognormal ( $q=1.2)$ channel capacity vs Average SNR corresponding to different range of $\sigma_{d B}$.

\section{Conclusion}

The characterization of extreme fluctuations over shadowing phenomena due to random obstacles in wireless propagation channels is essential for the evaluation of various performance metrics. The proposed $q$-Lognormal model well characterized these fluctuation phenomena in various fading environments than the conventionally used shadowing models. The proposed model operates well with the synthesized fading data in the continuous range of real valued Tsallis' parameter $q, 1<q<3$. Furthermore, the adaptability of the presented $q$-Lognormal model in characterizing the shadowing phenomena have been validated using different statistical tests viz., chi-square test and symmetric JS measure. Furthermore, the theoretical model for average channel capacity in slow fading environments is obtained corresponding to the proposed $q$-lognormal model.

It is profound that the $q$-Lognormal model adapts to varying fading scenarios in the real valued domain of Tsallis' parameter ' $q$ ' and also mimics various well-known shadowing models, which explicitly reinforced the computation of higher dimensional performance metrics viz., CDF with respect to channel capacity in terms of the well-known Gauss Hypergeometric function $\left({ }_{2} F_{1}().\right)$, evaluation of generalized $k_{t h}$ moments, the relative fluctuations i.e., $\mathrm{CV}$ over the decibel spread. These measures have been computed and are found to be in closed agreement with the simulation results. 


\section{References}

1. Simon, M. K., \& Alouini, M. S. (2005). Digital communication over fading channels (Vol. 95). John Wiley \& Sons.

2. Hansen, F., Meno, F.I.: 'Mobile fading rayleigh and lognormal superimposed', IEEE Transactions on Vehicular Technology, 1977, 26, (4), 332-335

3. Coulson, A. J., Williamson, A. G., Vaughan, R. G.: 'A statistical basis for lognormal shadowing effects in multipath fading channels', IEEE Transactions on Communications, 1988, 46, (4), 494-502.

4. Shankar, P. M. (2017). Fading and shadowing in wireless systems. Springer.

5. Rappaport, T. S. (1996). Wireless communications: principles and practice (Vol. 2). New Jersey: prentice hall PTR.

6. Abramowitz, M., Stegun, I. A., \& Romer, R. H. (1988). Handbook of mathematical functions with formulas, graphs, and mathematical tables.

7. Bebortta, S., Singh, A. K., Mohanty, S., \& Senapati, D. (2020). Characterization of range for smart home sensors using Tsallis entropy framework. In Advanced computing and intelligent engineering, Springer, Singapore, 265-276.

8. Singh, R., Soni, S. K., Raw, R. S., \& Kumar, S. (2017). A new approximate closed-form distribution and performance analysis of a composite Weibull/log-normal fading channel. Wireless Personal Communications, 92(3), 883-900.

9. Senapati, D. (2016). Generation of cubic power-law for high frequency intra-day returns: Maximum Tsallis entropy framework. Digital Signal Processing, 48, 276-284.

10. Tsallis, C., \& Mechanics, N. E. S. (2004). Construction and Physical Interpretation. Nonextensive Entropy Interdisciplinary Applications, 1-52.

11. Holtzman, J. M. (1992). A simple, accurate method to calculate spread-spectrum multiple-access error probabilities. IEEE Transactions on Communications, 40(3), 461464 .

12. Namaki, A., Lai, Z. K., Jafari, G. R., Raei, R., \& Tehrani, R. (2013). Comparing emerging and mature markets during times of crises: A non-extensive statistical approach. Physica A: Statistical Mechanics and its Applications, 392(14), 3039-3044.

13. Goldsmith, A. (2005). Wireless communications. Cambridge: Cambridge university press.

14. Abe, S., \& Bagci, G. B. (2005). Necessity of q-expectation value in nonextensive statistical mechanics. P hysical Review E, $71(1), 016139$.

15. Das, S., \& Bhattacharya, A. (2020). Application of the Mixture of Lognormal Distribution to Represent the First-Order Statistics of Wireless Channels. IEEE Systems Journal, 14(3), 4394-4401.

16. Shankar, P. M. (2011). Statistical models for fading and shadowed fading channels in wireless systems: A pedagogical perspective. Wireless Personal Communications, 60(2), 191-213.

17. Mukherjee, T., Pati, B., \& Senapati, D. (2021). Performance Evaluation of Composite Fading Channels Using q-Weibull Distribution. In Progress in Advanced Computing and Intelligent Engineering, Springer, Singapore, 317-324.

18. Chauhan, P. S., Tiwari, D., \& Soni, S. K. (2017). New analytical expressions for the performance metrics of wireless communication system over Weibull/Lognormal composite fading. AEU-International Journal of Electronics and Communications, 82, 397-405.

19. Shannon, C. E. (2001). A mathematical theory of communication. ACM SIGMOBILE Mobile Computing and Communications Review, 5(1), 3-55.

20. Laourine, A., Stephenne, A., \& Affes, S. (2009). On the capacity of log-normal fading channels. IEEE Transactions on Communications, 57(6), 1603-1607.

21. Sagias, N. C., Zogas, D. A., Karagiannidis, G. K., \& Tombras, G. S. (2004). Channel capacity and second-order statistics in Weibull fading. IEEE Communications Letters, 8(6), 377-379.

22. El Bouanani, F., Ben-Azza, H., \& Belkasmi, M. (2012). New results for Shannon capacity over generalized multipath fading channels with MRC diversity. EURASIP Journal on Wireless Communications and Networking, 2012(1), 336.

23. Mukherjee, T., Singh, A. K., \& Senapati, D. (2019). Performance Evaluation of Wireless Communication Systems over Weibull/q-Lognormal Shadowed Fading Using Tsallis Entropy Framework. Wireless Personal Communications, 106(2), 789-803. 
24. Foerster, J. (2002). Channel modelling sub-committee report final. IEEE P802. 1502/368rs-SG3a.

25. Alouini, M. S., \& Goldsmith, A. J. (1999). Area spectral efficiency of cellular mobile radio systems. IEEE Transactions on vehicular technology, 48(4), 1047-1066.

26. Nayak, G., Singh, A. K., \& Senapati, D. (2020). Computational Modeling of NonGaussian Option Price Using Non-extensive Tsallis Entropy Framework. Computational Economics, 1-19.

27. Mukherjee, T., Pati, B., \& Senapati, D. (2019) Performance Evaluation of Composite Fading Channels Using q-Weibull Distribution. In Progress in Advanced Computing and Intelligent Engineering, 317-324. Springer, Singapore.

28. Singh, A. K., Senapati, D., Mukherjee, T., \& Rajput, N. K. (2019). Adaptive Applications of Maximum Entropy Principle. In Progress in Advanced Computing and Intelligent Engineering, 373-379. Springer, Singapore.

29. Khandelwal, V. (2011). MGF and high order moment of channel capacity in log-normal fading environment. In Proceedings of the 1st International Conference on Wireless Technologies for Humanitarian Relief, 99-106. 
Figures

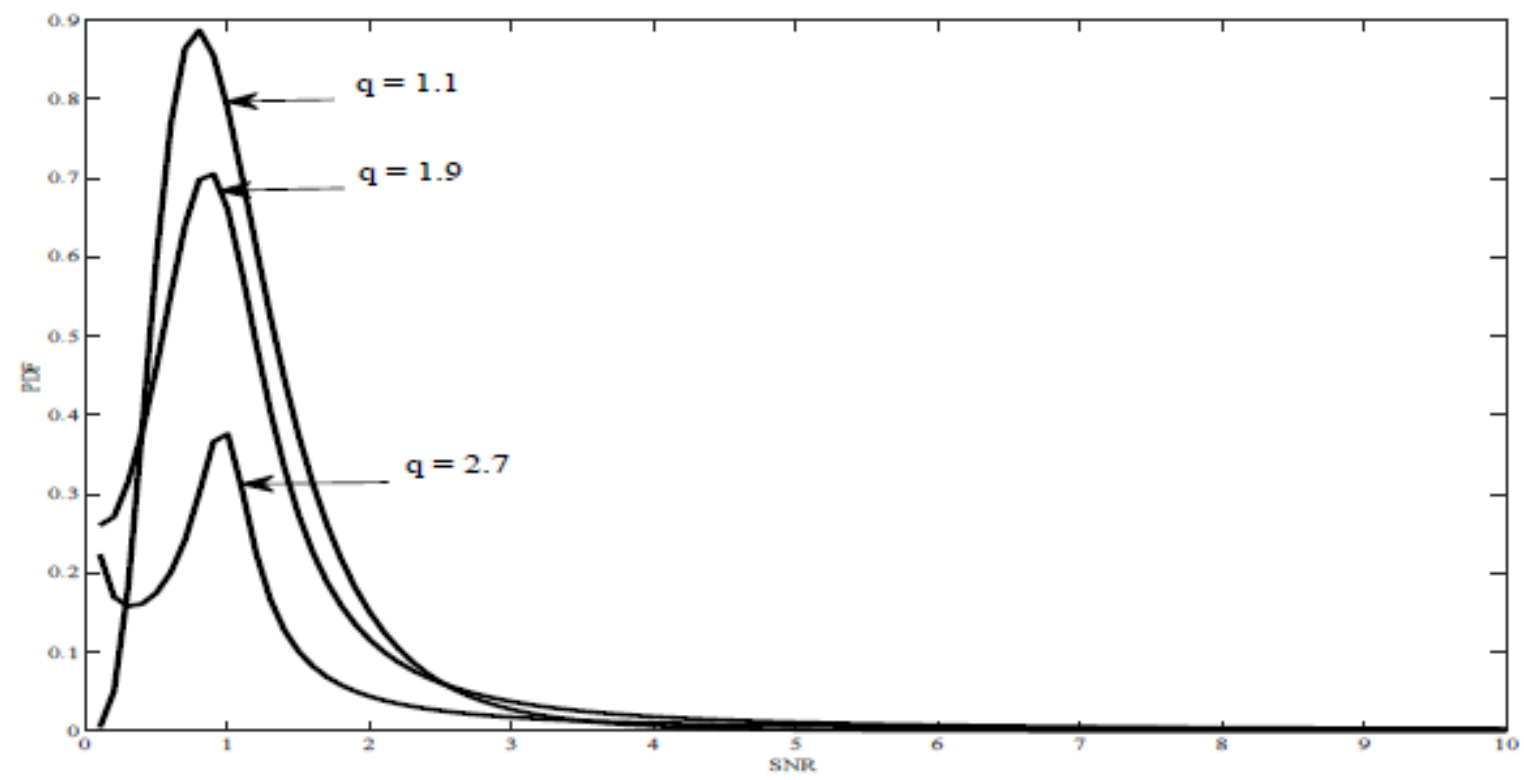

Figure 1

Illustration of q-Lognormal model of SNR $(\mathrm{Y})$ with respect to different values of parameter q.

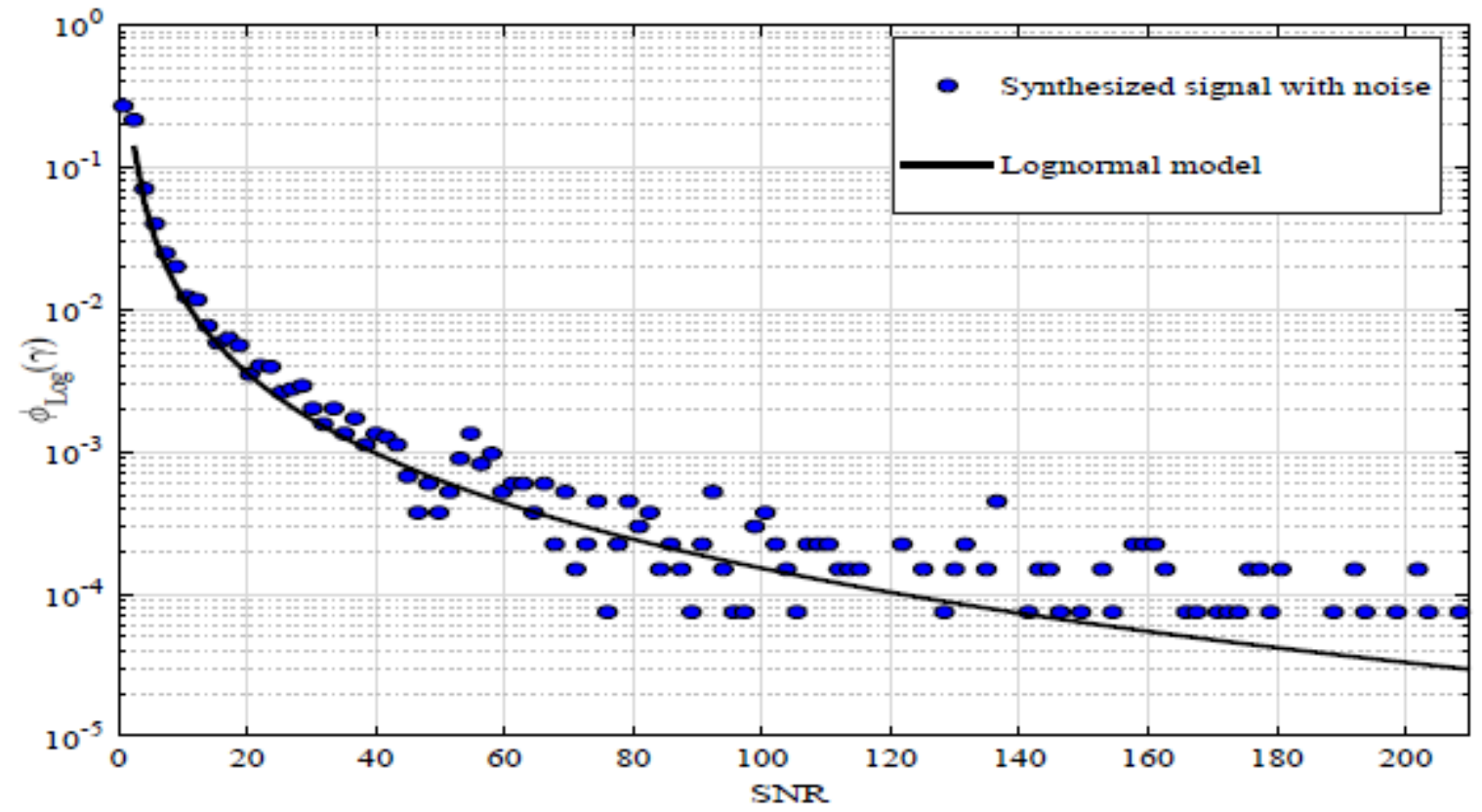

Figure 2

Illustration of log-normal model corresponding to synthesized signal. 


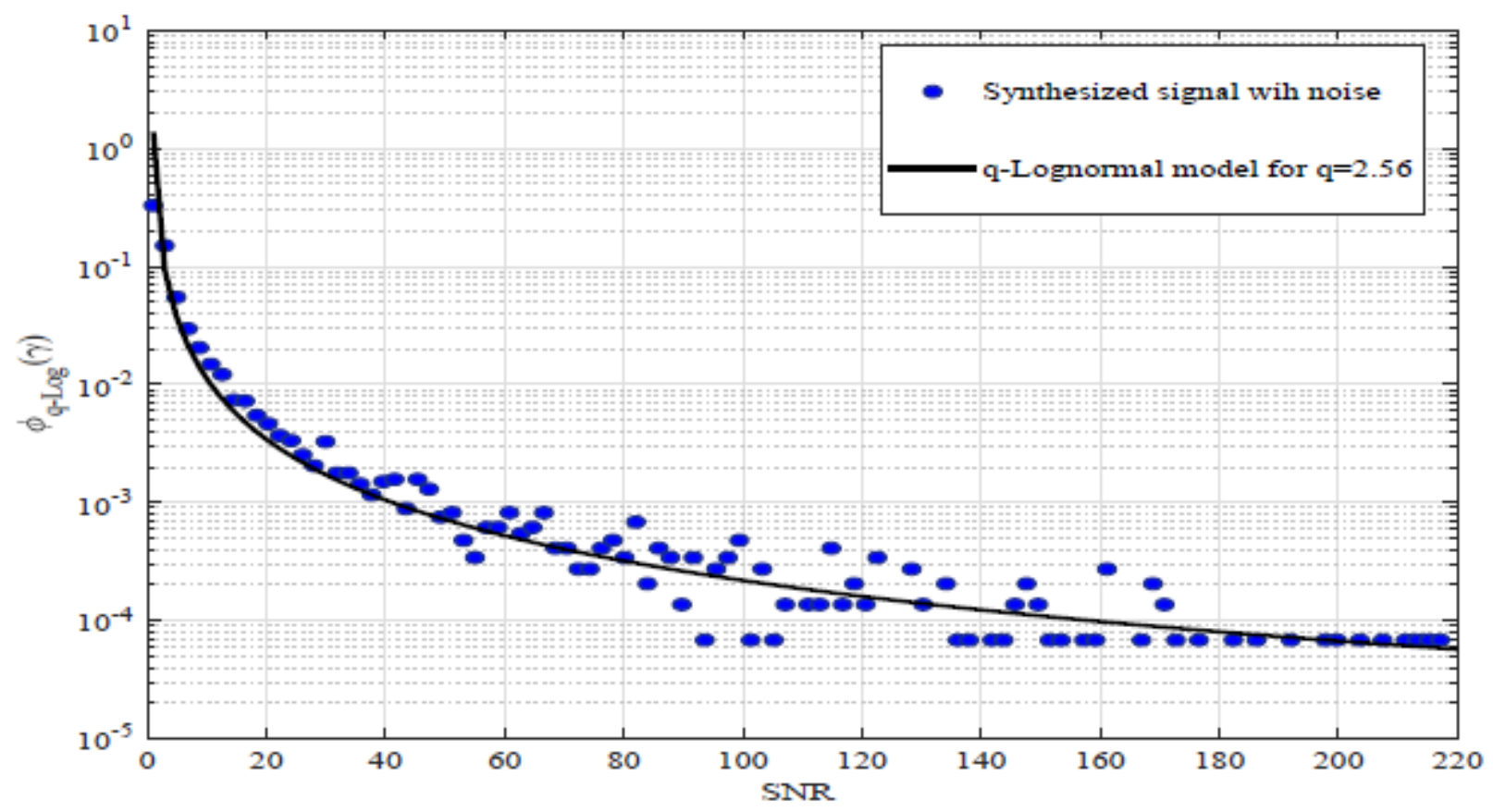

\section{Figure 3}

Illustration of q-Lognormal model with respect to synthesized signal for $\mathrm{q}=2.56$.

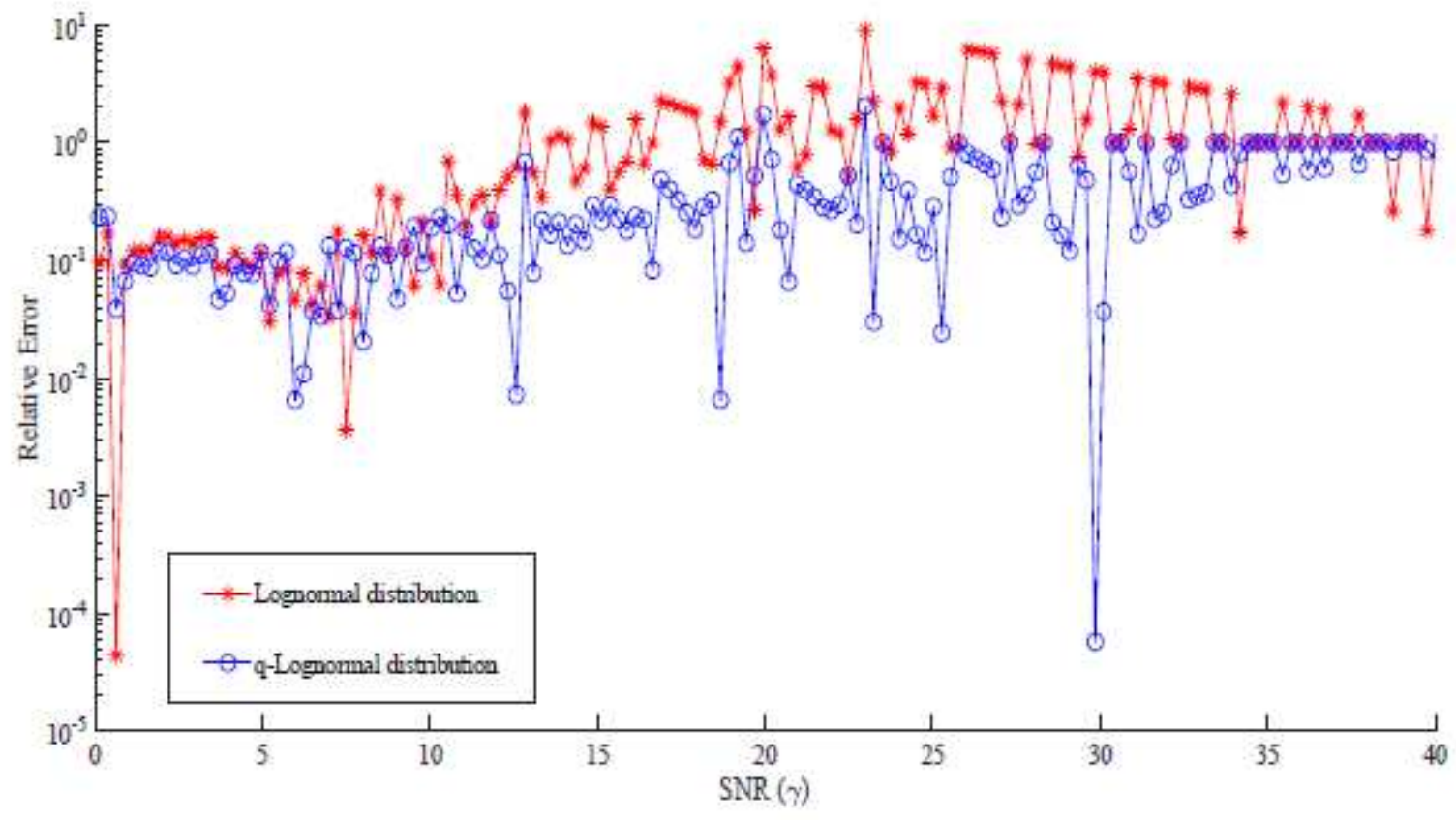

\section{Figure 4}

Comparison of Relative error of q-Lognormal model in contrast to conventional Lognormal distribution. 


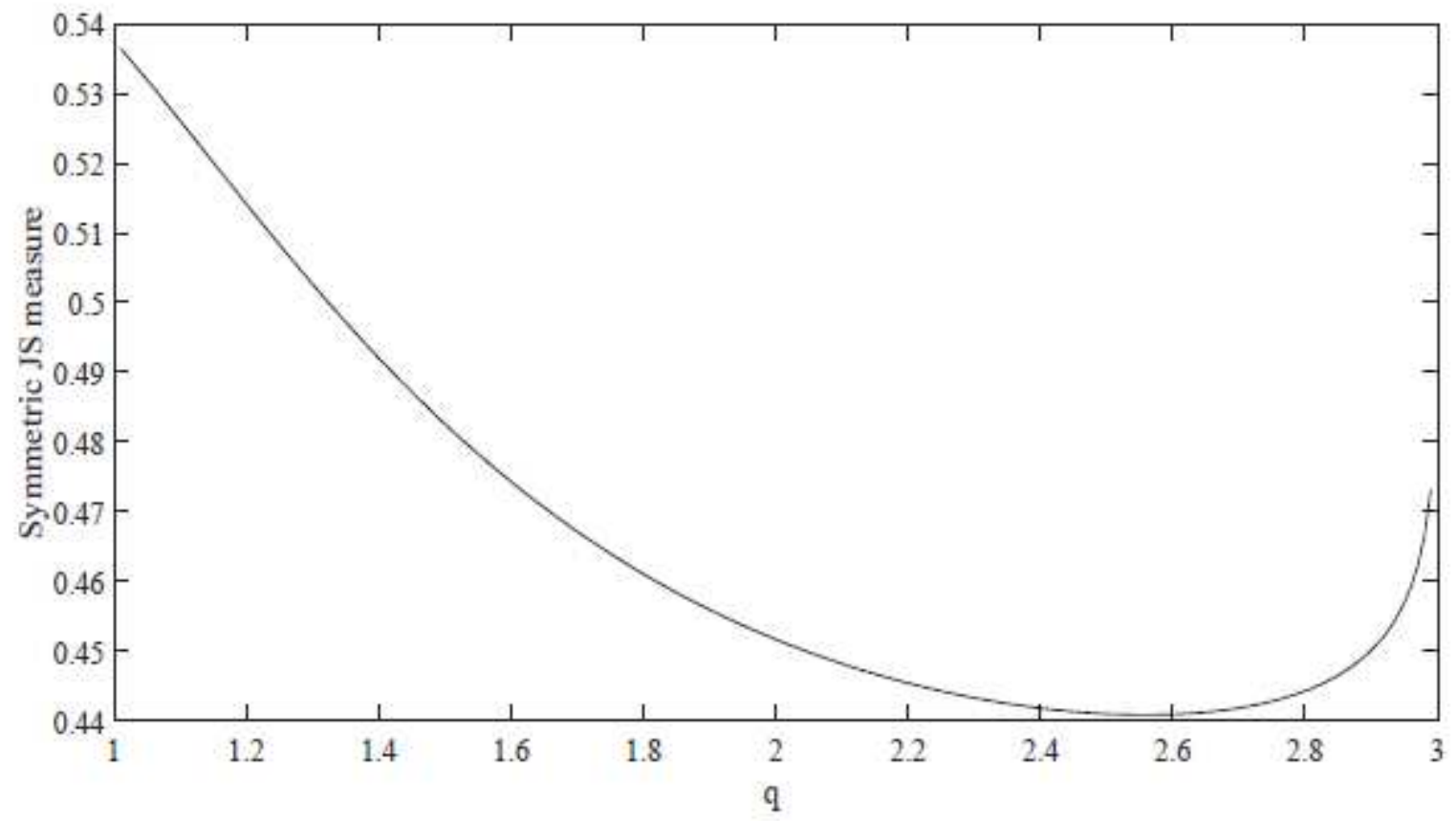

Figure 5

Symmetric JS measure for q-Lognormal model and synthetic signal of $\mathrm{O}(108)$ cor- responding to $\mathrm{q}$.

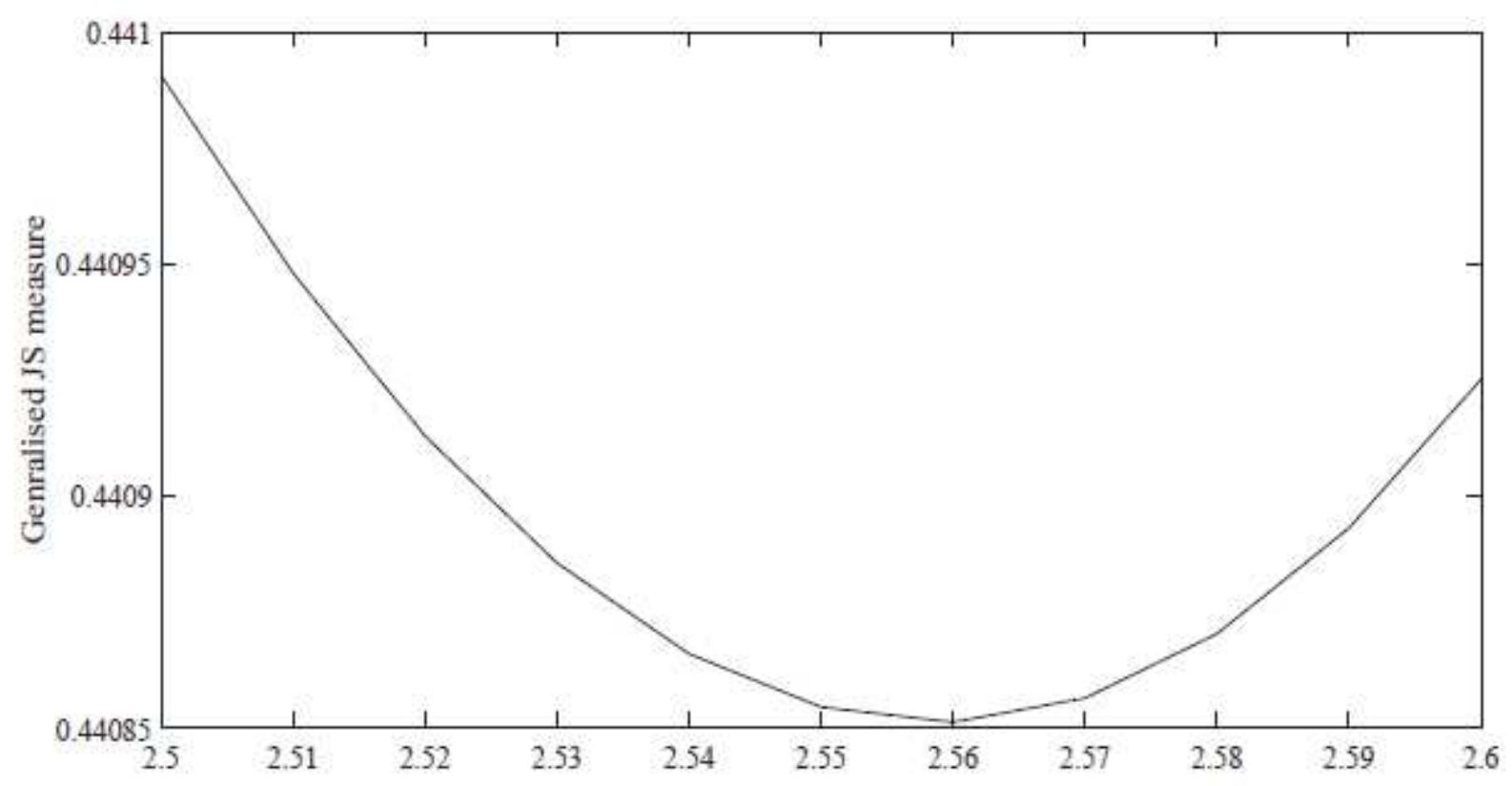

Figure 6

Cropping of Fig.5 in the close neighborhood of $q=2: 5$. 


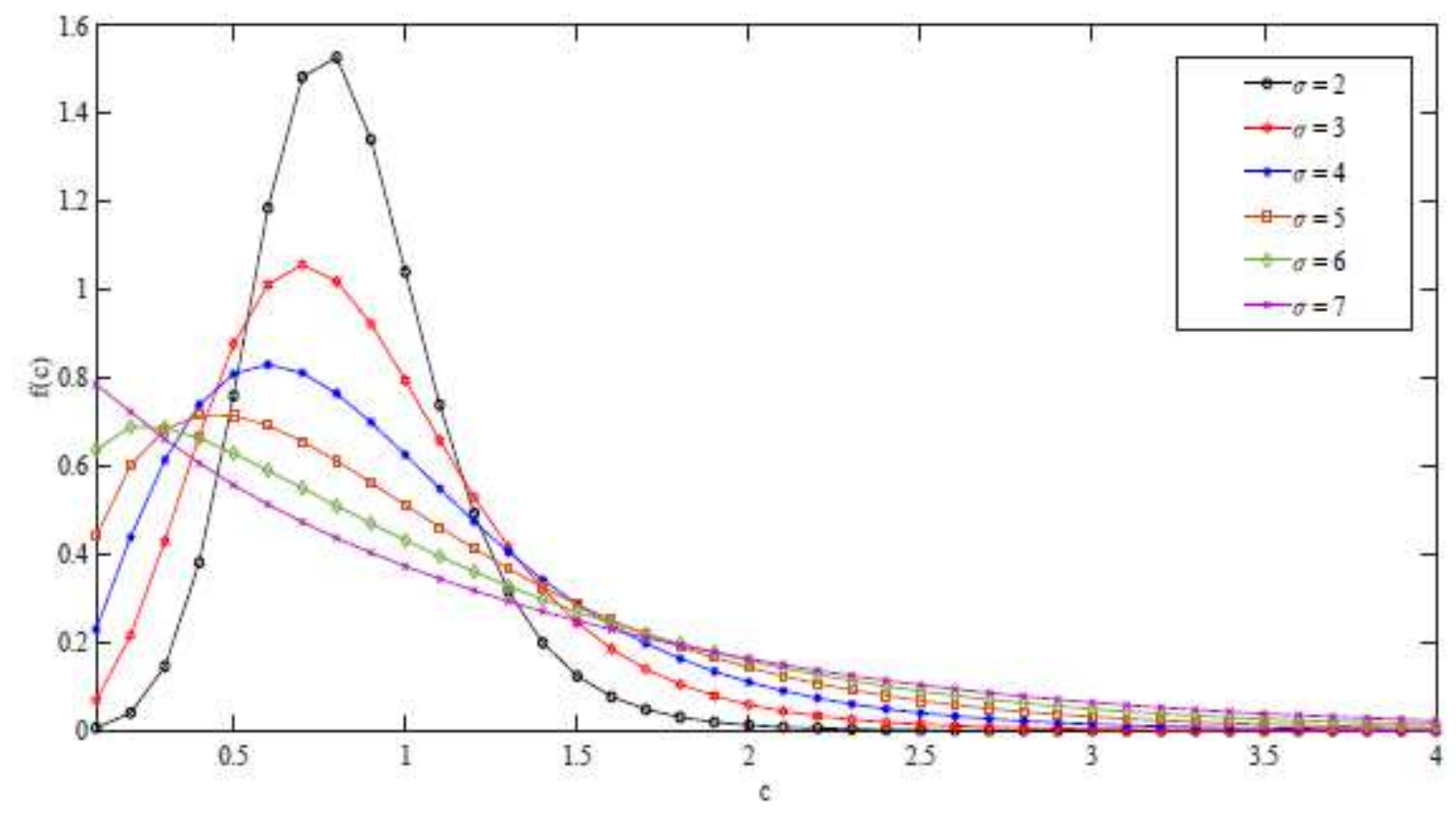

Figure 7

PDF of q-Lognormal channel capacity over $q=1.2, \mu \mathrm{dB}=1$ with different range of $\sigma \mathrm{dB}$.

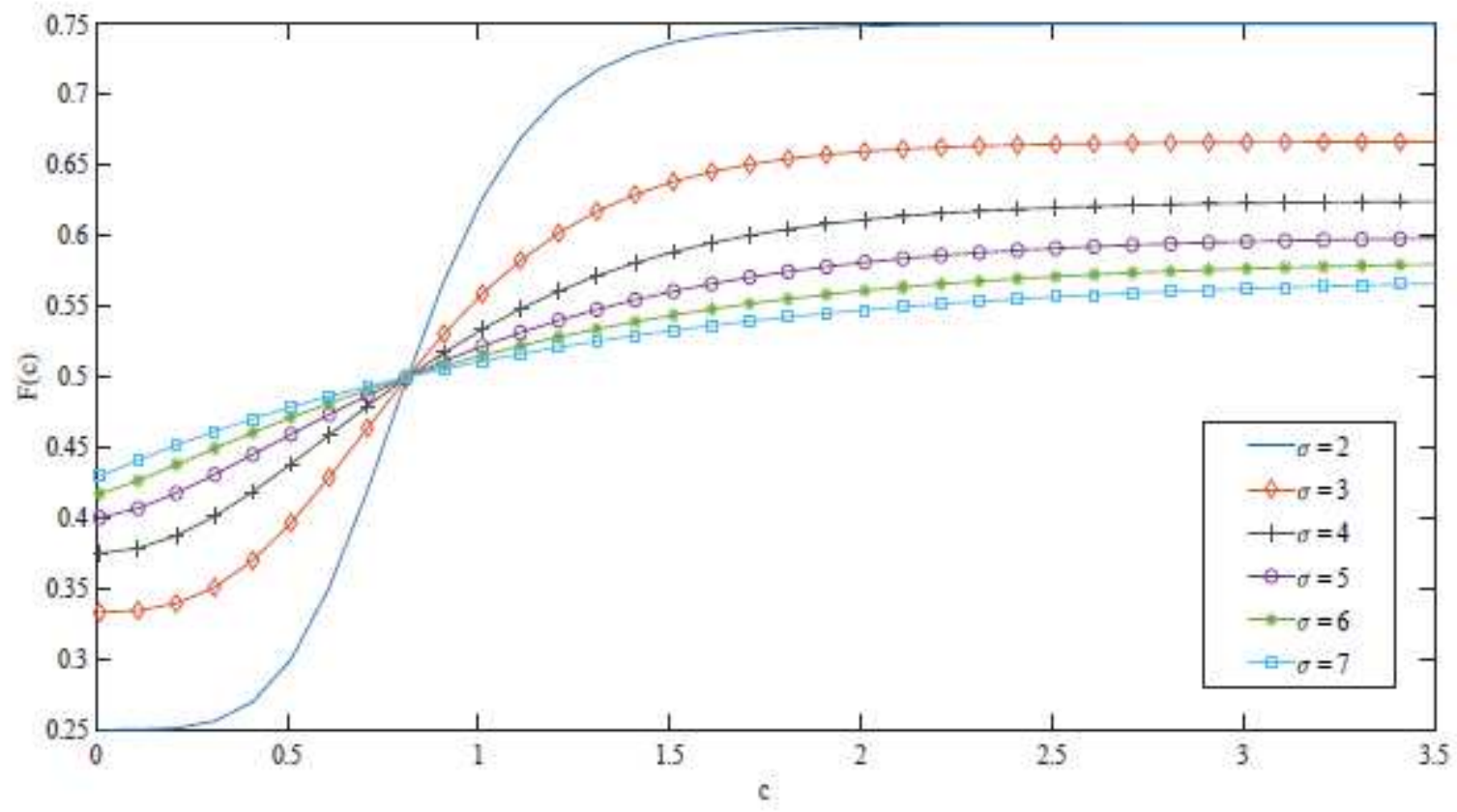

Figure 8

CDF of $q$-Lognormal channel capacity over $q=1.2, \mu \mathrm{dB}=1$ with different range of $\sigma \mathrm{dB}$. 


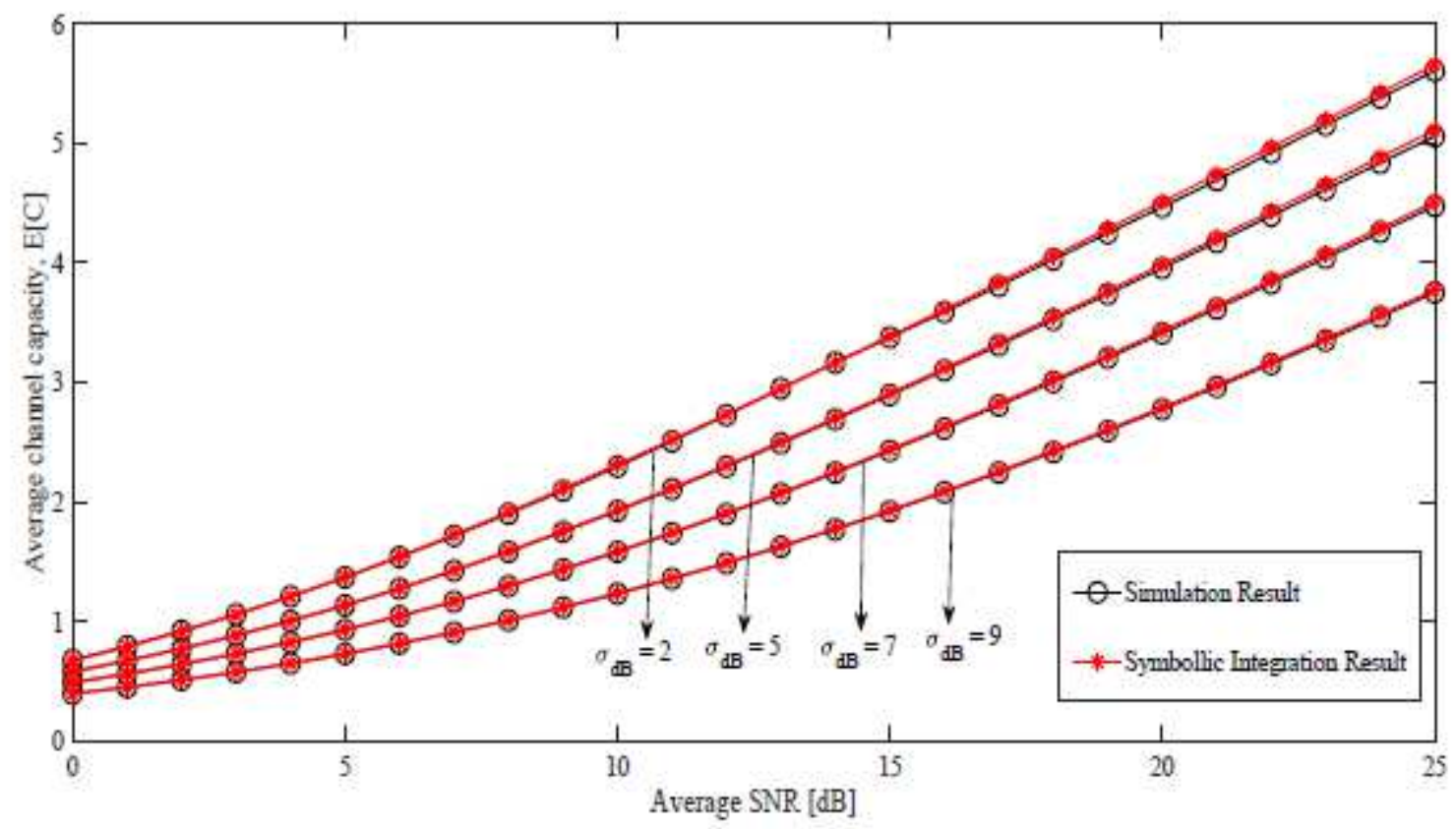

Figure 9

Plot of rst moment of Average channel capacity $(E[C])$ over q-Lognormal model $(q=1.2)$ corresponding to different $\sigma \mathrm{dB}$.

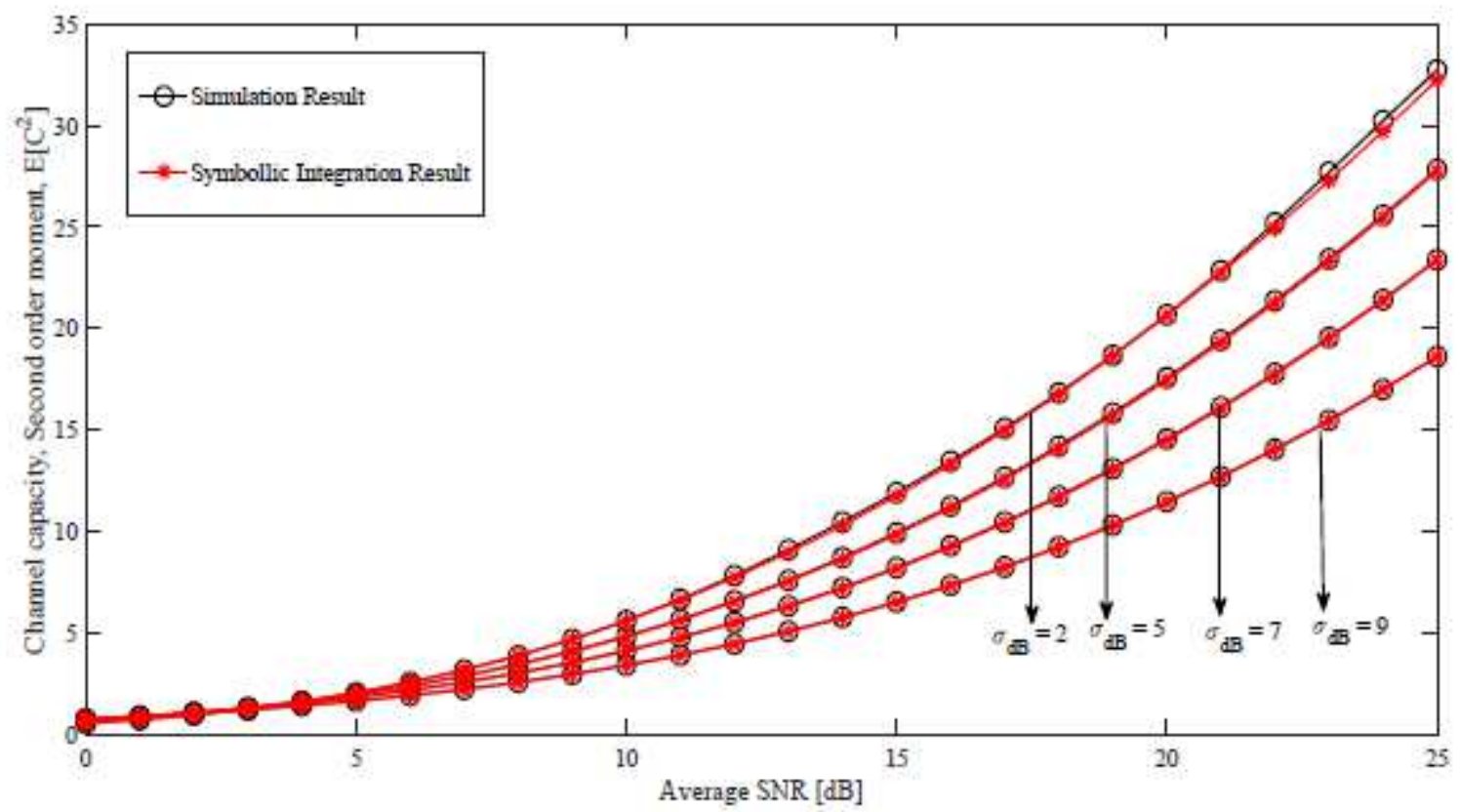

Figure 10

Plot of second moment of channel capacity $(E[C 2])$ over $q$-Lognormal model $(q=1.2)$ corresponding to different $\sigma d B$. 


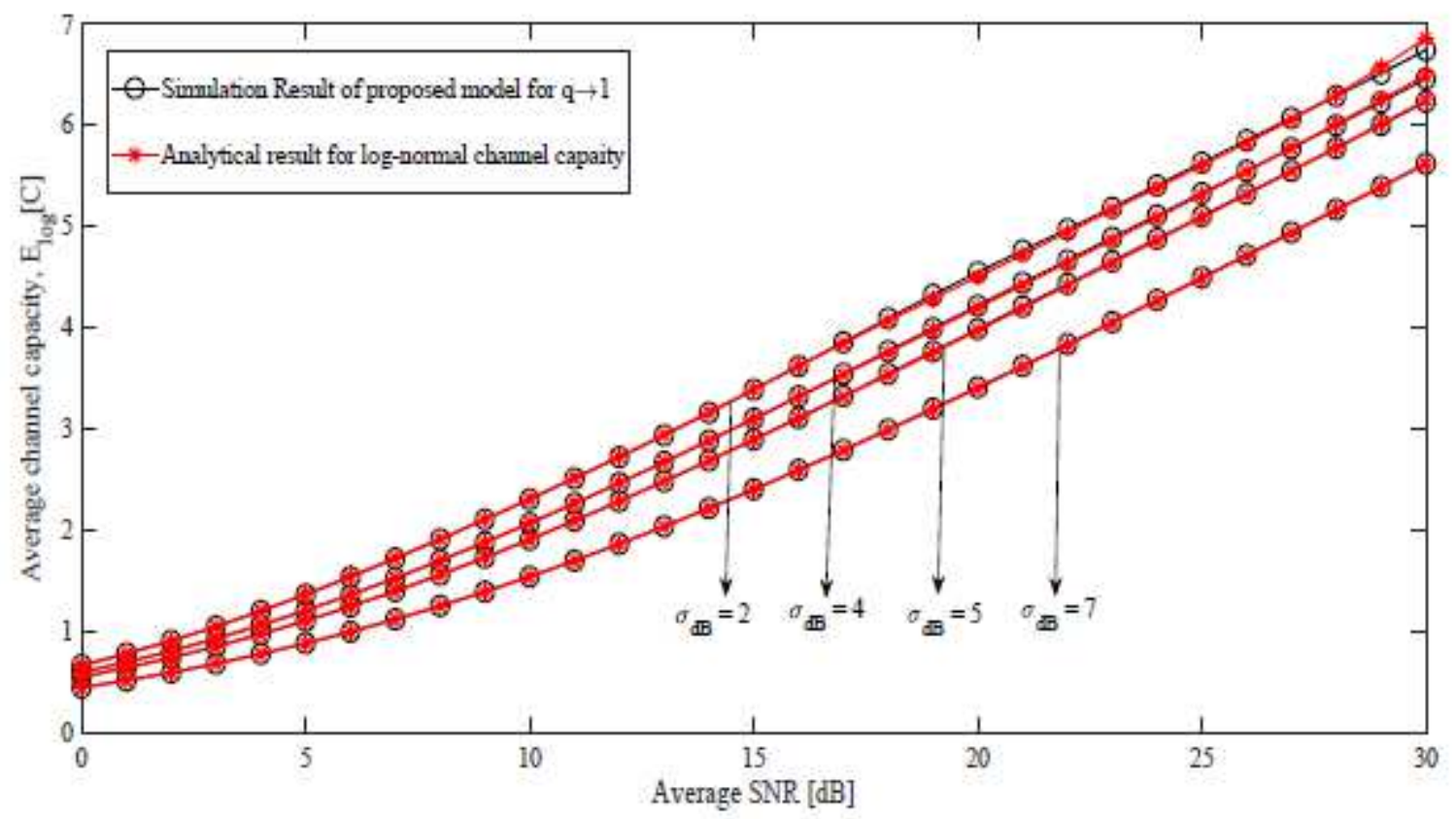

Figure 11

Plot of rst moment of log-normal and q-Lognomal (q $\otimes 1$ ) channel capacity vs Average SNR corresponding to different range of $\sigma \mathrm{dB}$.

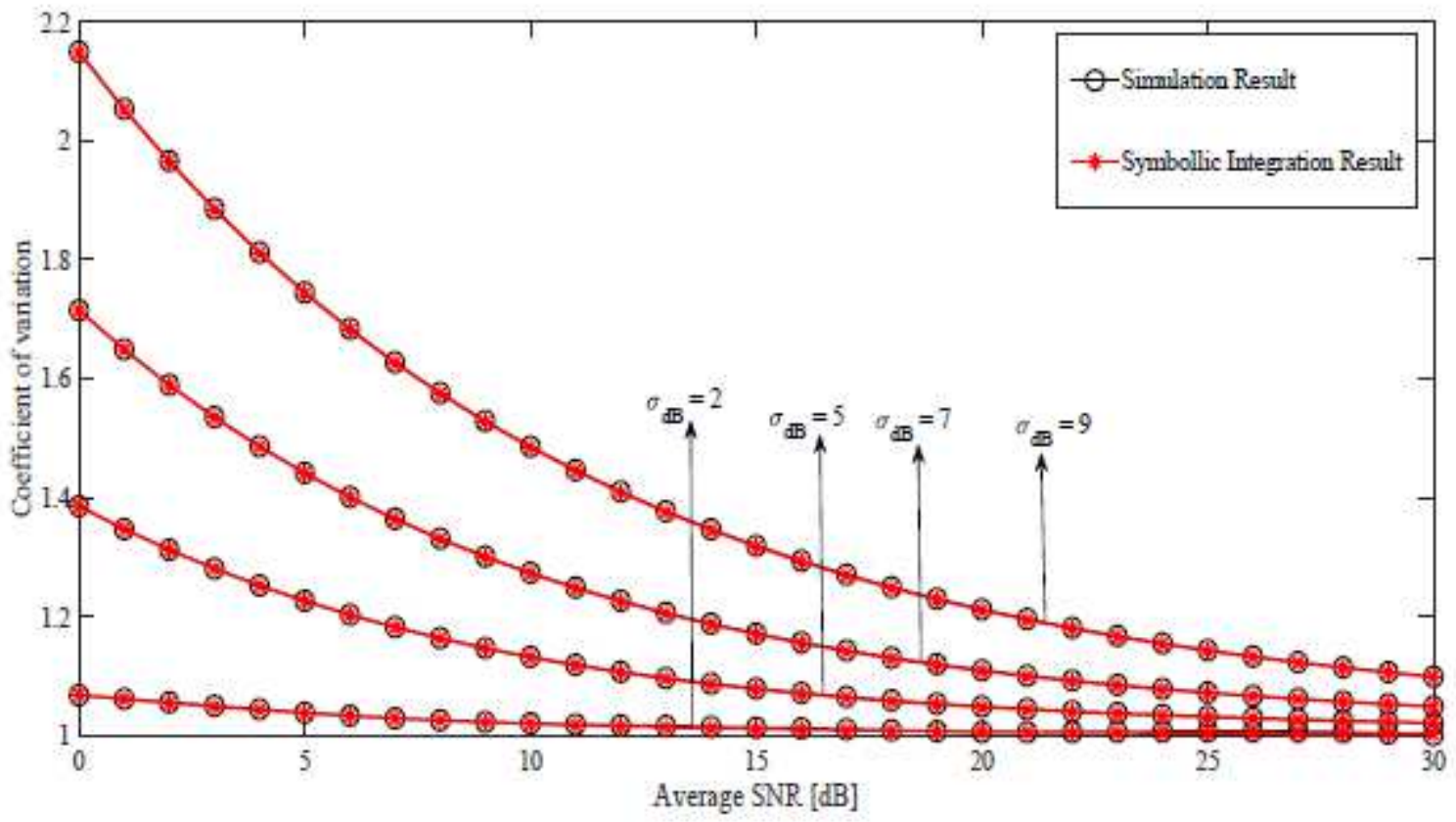

Figure 12

Plot of CV for q-Lognormal $(q=1.2)$ channel capacity vs Average SNR corresponding to different range of $\sigma \mathrm{dB}$. 